\title{
AN EXPERIMENTAL STUDY OF THE CORONA VOLTMETER
}

\author{
By H. B. Brooks and F. M. Defandorf
}

\section{ABSTRACT}

Whitehead's corona voltmeter (rating $140 \mathrm{kv}$ effective voltage) was modified in order to improve control of pressure, temperature, and humidity. A new and highly accurate method of determining the 60-cycle crest voltage was used in studying this device, within a pressure range of three atmospheres and a temperature range of $15^{\circ}$ to $45^{\circ} \mathrm{C}$. Twelve corona rods of diameters from 0.12 to $1.1 \mathrm{~cm}$ were used in this investigation. One outer cylinder of $25 \mathrm{~cm}$ diameter was used.

Increase in humidity was found to lower the corona voltage for a dusty or otherwise soiled rod, although it generally caused an increase for carefully cleaned rods.

Maintenance and duplication of a given condition of the surface of the corona rod appear to be the factors that limit the repeatability in the present type of instrument. Further investigations of the effect of surface conditions would be of interest.

Our experimental results have led us to propose the following modified law of corona formation in dry air between concentric cylinders:

$$
g=A \delta+B \sqrt{\delta / r}-C / r
$$

for which our experiments gave

$$
A=27.95 \quad B=11.18 \quad C=0.365
$$

A nomogram has been devised with which the crest voltage for a given set of rods, pressure, and temperature may be rapidly determined without any calculation. This particular nomogram has been laid out on the basis of dry air.

The appendix contains a discussion of the relative effects of observational errors in the measurement of air pressure, air temperature, and the radii of the rod and the cylinder.

\section{CONTENTS}

I. Introduction

1. Historical development

2. Corona formation

II. Description of apparatus and methods_._. 597

1. Alterations in the corona voltmeter.......... 597

2. Temperature measurement_._. 601

3. Pressure measurement__._. 603

4. Determination of humidity

5. Detection of corona formation _... 604

6. Temperature control___._. 606

7. Procedure in cleaning rods_._. 606

III. Production and measurement of voltage

1. Sources of voltage

2. Measurement of effective voltage _._._._. 607

3. Determination of wave form and crest factor......... 610 $111896^{\circ}-28-1$ 
IV. Observations on the corona voltmeter

1. Description of observers' duties

2. Sources of error

612

613

V. Results

1. Method of presenting data

615

2. Effect of contamination of rod surface

615

3. Effect of humidity

616

4. Effect of change in temperature

618

5. Empirical equation to fit observations

6. A nomogram that simplifies voltage determination

VI. Advantages and disadvantages of the corona voltmeter and suggestions for its improvement

VII. Acknowledgments

VIII. Appendix

1. Effect of errors in observed data

2. References

\section{INTRODUCTION}

\section{HISTORICAL DEVELOPMENT}

H. J. Ryan $(1)^{1}$ originally proposed the use of corona as a crestvoltage indicator. Later, J. B. Whitehead $(2,3)$, after a careful study of the electric strength of air, suggested the initial formation of corona on clean, round wires as a means for the accurate determination of crest voltage. $\mathrm{He}$ and his coworkers (3) subsequently devised and studied instruments making use of this phenomenon and called them corona voltmeters.

Such an instrument consists of two concentric cylinders-one, an inner wire or rod, and the other, an outer inclosing cylinder usually grounded. The inner rod, which is insulated, is connected to the conductor whose crest voltage with respect to ground it is desired to determine. The cylinder and the rod are contained in a metallic inclosure so designed that the pressure and temperature of the inclosed air may be controlled. The initial formation of corona may be detected by the sound, or light, or ionization that accompany it.

Many investigations of the phenomena of corona have been made, and an empirical law called the "Law of corona" has been developed by Whitehead, F. W. Peek (4), and others, which gives the electric gradient at which the initial formation of corona occurs as a function of pressure, temperature, and radii of the cylinders. The law, as it has been developed, may be written

$$
g=A \delta+B \sqrt{\delta / r}
$$

\footnotetext{
1 The figures given in parentheses here and throughout the text relate to the reference numbers given at the end of this paper.
} 
wherein $g$ is the gradient in volts per centimeter calculated on the basis of potential theory to exist at the surface of the rod in the absence of space charge and contact potential, at which corona begins to form at the surface of the inner cylinder or rod. In the case of concentric cylinders

$$
g=\frac{V_{\mathrm{c}}}{r \log _{\mathrm{e}}(R / r)}
$$

in which $V_{\mathrm{e}}$ is the crest voltage between the outer cylinder and the rod at which corona begins to form and $r$ and $R$ are the radii in centimeters of the rod and outer cylinder, respectively. $\delta$ is called the density factor by Peek. It is defined by

$$
\delta=\frac{3.921 P}{T}=\frac{3.921 P}{273.1+t}
$$

in which $P$ is the absolute pressure in centimeters of mercury reduced to $0^{\circ}$ C., and $T$ is the absolute temperature in degrees centigrade. $\delta$ is unity for standard conditions of $P=76 \mathrm{~cm}$ of mercury at $0^{\circ} \mathrm{C}$., and $t=25^{\circ} \mathrm{C}$.

The law of corona as given by equation (1) is the result of the work of Whitehead and of Peek. While agreeing as to the form of the relation these investigators have differed somewhat as to the numerical values of the coefficients $A$ and $B$. It seems probable that the discrepancies are the result of the necessarily approximate methods heretofore available for the measurement of high voltages. However, for a given apparatus the nature of the imperfection is that of a constant relative error of unknown magnitude, and hence it has not prevented the establishment of the facts concerning the definitely reproducible formation of corona and the determination of suitable experimental means for detecting it.

At the time this investigation was planned the sphere and needle gaps were both used as standard methods of measurement of crest voltage in accordance with A. I. E. E. specifications. The work of Whitehead and his associates indicated that the corona voltmeter had the following advantages: (a) It repeats its indications exceptionally well, $(b)$ its power consumption is low and transient effects are correspondingly negligible, and $(c)$ since the corona electrodes are ordinarily inclosed in a grounded cylinder, measurements are not affected by the proximity of surrounding objects. These arguments indicated that a higher accuracy of measurement might be expected with the corona voltmeter than with either the needle or sphere gap and led to the work described in this paper. The instrument used was the large corona voltmeter (rating $140 \mathrm{kv}$ effective value) which Whitehead and Isshiki had calibrated with considerable care over the greater portion of its useful range. 
On summarizing earlier experimental work on alternating-current corona it was found that considerable data had been accumulated using air, other mixed gases, and pure gases within quite wide ranges of pressure and temperature, with various diameters of rods and outer cylinders, for both continuous and alternating voltage; and it was found that Whitehead alone (and in one of his earliest papers) presents quantitative studies of the effect of moisture in air on initial-corona alternating voltage and that his results as to the effect of water vapor were negative. Some other investigators took precautions to eliminate the moisture content of their gases by using various drying processes but showed no measurements of moisture content and its effect on the alternating voltage necessary to produce corona. Farwell (5) showed that moisture content affected continuous-voltage corona in air. The present investigation includes a quantitative study of the effect of humidity.

The objects of the present investigation were to determine with greater accuracy the electric strength of air at the surface of round rods, to determine how this property of air depends on humidity, and to facilitate the practical use of the corona voltmeter for crestvoltage measurements. An incidental object of the investigation has been to simplify the computation of the crest voltage at the terminals of the corona voltmeter, in order to facilitate its use as a practical instrument for crest-voltage measurements, and to evolve a graphical means for avoiding all computation, as would be highly desirable for industrial applications of the instrument. In this connection it was considered desirable to investigate the effects on the accuracy of measurements of small relative errors in the observed data. Results of these investigations appear in the appendix.

While considerable theoretical interest attaches to the question of the corona-forming intensities of positive and negative continuous voltages, and the relation between them and the intensity for alternating voltages, the immediate engineering need for more accurate information concerning the latter was felt to be much the greater, and the present investigation has, therefore, been limited to alternating voltages of practically sine wave form and of 60 cycles frequency.

\section{CORONA FORMATION}

The ideal corona voltmeter would consist of two perfectly smooth, clean, concentric metallic cylinders between which the voltage is applied, separated by a layer of pure simple gas. The precision of such a corona voltmeter should depend only upon the repeatability of initial formation of corona and upon the adjustable pressure and temperature of the gas between the concentric cylinders. Since other variables supposedly are absent, we need only to define initial formation of corona before studying the effect of changes in the two vari- 
ables, pressure and temperature, on the corona voltage. The name corona suggests that the phenomenon is a visible one and implies that it should be detected visually. However, the light emitted upon the initial formation of corona by a layer near the surface of the rod is too faint to be detected except in a darkened space and with rested eyes. As the voltage between the cylinders is raised the light emitted and the depth of the emitting layer at the coronaforming surface may be seen in the dark to gradually increase as voltage is raised above the initial corona voltage. Simultaneously with the emission of light, ionization may be detected and a characteristic sound produced by the corona may be heard. As Whitehead has shown, either of these latter methods of detection of initial formation of corona seems more readily adaptable for ordinary laboratory use than the visual method. Furthermore, Whitehead and others have shown that the aural, visual, and ionization methods of detection agree closely. It was customary in the work here described to depend upon the aural method for recorded observations and to check the simultaneity of detection, using the ionization method for each run. For any one of the three methods of detection a sudden definite increase in signal intensity from a practically zero value is taken as the indication of initial formation of corona.

When a continuous voltage of moderate value is applied between a pair of electrodes surrounded by a gas at approximately atmospheric pressure and room temperature, the resulting current through the gas consists merely of a feeble convection of charge by the normal ionization which researches on atmospheric electricity have shown to exist at all times as a result of radioactive material in the earth and of cosmic radiation. This current is of the order of $10^{-15} \mathrm{am}-$ pere, and when the applied voltage is 200 to 500 volts it reaches a saturation value fixed by the source of ionization. For this condition the space charges present in the space between the electrodes are quite negligible in their effect on the electric-field distribution.

As the voltage is increased a condition is finally reached at which the velocity acquired by such of the negative ions as are electrons, during an unusually long free path, is occasionally sufficiently great to enable them to ionize the molecules which they strike at the end of their path. Such ionization by collision has been studied in detail by Thomson, Townsend, and others (6) and may result in a value of current many (perhaps $10^{4}$ ) times the original saturation current. A little consideration will show that this phenomenon may produce a measurable "dark current," which may even be sufficient to distort the electric field slightly by the resulting space charge. It will be noted, however, that the "progeny" of any given electron will necessarily originate at points of more positive potential than did their "parent." Hence, if the ionizing agency were completely cut 
off, all the ions would ultimately be swept out of the field and the current would cease. Also, the current flowing is necessarily still a continuous function of the applied voltage. An additional process is necessary to produce the abrupt change in régime which is characteristic of the beginning of a self-sustaining discharge, such as corona discharge or spark over.

Townsend makes the hypothesis (which we may call No. 1) that this additional process consists in the beginning of ionization by collisions between the positive ions and the neutral gas molecules; and such a process would, indeed, account for the observed phenomena. Other workers have questioned Townsend's assumption, however, and have suggested alternative hypotheses: No. 2, that the impact of the positive ions may eject electrons from the cathode; and No. 3, that radiation emitted during or after the ionizing collisions between electrons and molecules may exert a photoelectric effect on $(a)$ the gas molecules or $(b)$ the metal of the cathode.

Hypotheses No. 1 and No. 3 (a) would indicate that the discharge should depend on the gas only and be independent of the electrode material, and this was long thought to be the case. However, later work on the time lag in spark discharge and on spark over at low pressures or with impulses of short duration, indicates that the condition of the electrode surface does influence the discharge to some extent. The present work also indicates a similar effect. It therefore appears that the processes called for by hypothesis No. 2 or No. 3 (b) may also be occurring. In the case of discharges between a positive point and a plane, or, as in the present work, between a rod and a concentric cylinder, it is found that the discharge occurs when the electric-field intensity at the plane (or cylinder) is still far below that required for a discharge between planes or spheres. It is difficult to explain this fact on the basis of hypothesis No. 2, but No. $3(b)$ may still be applied.

Whatever hypothesis may be correct, it will be seen that any of them renders it possible for the process initiated by a single casual ion to yield a distribution of ions in which some may be situated even more favorably than the original one for producing other ions by collisions. When this stage is reached an unstable condition exists. The density of ions increases with great rapidity to relatively enormous values. When the electrodes have a radius of curvature large compared with their spacing-that is, the field is nearly uniform - this increase in density occurs throughout the space between them and a spark results. In this case the current flowing is limited almost entirely by the characteristics of the external circuit. On the other hand, in the case of points or of a slender wire concentric with a cylinder of relatively large radius, the great density of ionization is produced only in the region of intense field close to the point or wire. 
The electric carriers originating there may be sufficient to supply a current of a few milliamperes per meter of wire, and this current flows as a convection of charge across the annular volume between the corona glow and the outer cylinder. Some carriers recombine at a rate which increases with the ionic density, and these do not participate in the measured current flow. In any event, however, a state of equilibrium is reached for which the space charge present for this equilibrium condition serves to materially increase the field intensity close to the rod and to correspondingly reduce it in the outer parts of the field, thus making the transition, when instability is reached, even more abrupt.

As long as we assume that the ions resulting from this process are of equal size and mass independent of the sign of their charge and require an equal gradient for formation, we may expect the process of corona formation to be as outlined above. If, instead of continous voltage, alternating voltage is applied and gradually increased, an instantaneous value of voltage on the positive half wave is eventually reached at which ionization begins, the positive ions being repelled from the rod while the negative ions are attracted and partly neutralized at its surface. The voltage passes through a maximum value while corona continues to form and decreases to a value (somewhat higher than that for initial corona formation) at which corona formation ceases as a result of lowering of the gradient near the rod by space charge. The positive charge continues to move toward the outer cylinder for the rest of the positive half wave. When the voltage reverses, the positive ions reverse direction and move toward the rod and, because the direction of the force acting for this entire half wave is toward the rod, are almost completely neutralized. Since the rod is now negative, the positive-ion space-charge effect is such that it increases the gradient near the rod to a higher value than it would have at the same applied voltage in the absence of space charge. Therefore, on the negative half wave the ionizing gradient is reached earlier than it would be had the ionization of the positive half wave not preceded it. Consequently, negative corona begins to form earlier in the wave, or at a lower applied voltage, than it would in the absence of space charge. Those negative ions not neutralized by positive ions are forced toward the outer cylinder. As a result of recombination or neutralization of the incoming positive space charge by negative ions, the negative space-charge distribution is such that corona continues to form until a lower voltage is reached than that at which positive corona ceased. Similar effects are produced by the negative and positive space charges in succeeding half waves. After several cycles a steady state is reached which continues as long as the applied voltage is held constant, if the pressure and temperature remain the same. As a result of space charge the corona formation 
and cessation voltages for this steady state are slightly lower than the corresponding voltages for the first positive half wave for which corona began."

However, if the ionization gradient is lower for positive corona than for negative corona, then for a voltage just sufficient to produce positive corona on the following negative half wave all positive charges reach the rod and are neutralized, since they form at the crest of the positive half wave, travel outward for the rest of the positive portion of the wave, and are then forced back toward the rod during the complete negative half wave. If the mass of the ion increases (mobility decreases) due to association of other molecules, the ions may not reach the rod. In any event, the positive ions, before either reaching the rod while it is still negative or disappearing through recombination, produce a space-charge effect. If the positive ions have not increased in size before the crest of the negative half wave is reached, their effect is to increase the gradient near the rod, as they approach closer and closer to the rod. As the voltage is increased to higher values the combined effect of returning space charge and applied voltage is also to produce the ionizing gradient for negative corona before maximum a. c. voltage equivalent to negative d. c. corona voltage is reached. The negative ions move in a direction opposite to that of the positive ions which they do not neutralize and produce an effect, although of opposite sign, similar to that of the positive ions. Actually, the positive and negative spacecharge effects are of unequal magnitude, as reference to experimental observations to be given shortly will show.

The preceding discussion assumes that discharge forms for alternating voltage applied to the corona voltmeter as soon as the maximum instantaneous voltage reaches a value corresponding to the minimum voltage for initial formation of positive or negative corona for continuous voltages. Using the electroscope for detecting ionization resulting from corona formation, Whitehead and Brown (3) observed that initial formation of a. c. corona coincided with negative d. c. corona, which occurs at a higher value of voltage than positive $\mathrm{d}$. c. corona. Willis (7) working with a. c. corona on a cylindrical electrode about $1 \mathrm{~mm}$ in diameter, separated at adjustable distances from a plane wire-gauze electrode with a sensitive galvanometer and polarizing battery for detection of ions passing through the gauze electrode, found that the initial positive discharge or ionization current could not be detected at atmospheric pressure for separations greater than $8 \mathrm{~cm}$. For greater separations, the positive ions produced on the positive half wave supposedly return to the rod on the next or negative half wave, unless corona forms on the negative half wave, in which case space-charge effects alter the gradient so that positive ionization currents may be detected. For smaller 
separations some of the positive ions reach the gauze within the remainder of the positive half cycle. If the voltage is further increased, at separations less than $8 \mathrm{~cm}$ ionization sets in on the negative half wave, ${ }^{2}$ and its presence is indicated by a sudden break or increase in the positive ionization curve, of which the first portion is nearly a straight line. For separations greater than $8 \mathrm{~cm}$, at atmospheric pressure the straight portion of the curve is missing. At pressures somewhat below atmospheric with our larger spacing of $12 \mathrm{~cm}$ we have observed a corresponding break in the straight portion of the positive ionization-current curve. Willis has suggested that a corona voltmeter could be designed to use the straight portion of this curve that would permit very precise measurement of voltage, but unfortunately such a design would limit the range of the corona voltmeter to lower values than the present design of concentric cylinders with about $12 \mathrm{~cm}$ separation.

Our own observations gave the same initial corona-forming voltage for visual corona for either positive or negative half waves (observed with stroboscope) for the particular conditions under which visual observations were made. Simultaneous observations in this particular case, using also aural and ionization methods of detection, showed that the three methods gave the same corona-forming voltage within the limits of observation ( 0.02 per cent). These observations were made only on rod $D$ over a fairly restricted range of pressure (25 to $150 \mathrm{~cm} \mathrm{Hg})$. These results seem to support Willis's belief (7) that other observers of a. c. corona have for the most part observed the sudden increase in positive ionization and called it initial corona.

Apparently, in our case detection at atmospheric pressure consists in observing the light or noise incident to recombination of ions resulting from successive half waves, the one set returning to the rod and the other leaving, probably with a different velocity, while galvanometer detection usually corresponds to the beginning of negative corona which by its space-charge effect rapidly increases the positive ionization current which is observed.

\section{DESCRIPTION OF APPARATUS AND METHODS}

\section{ALTERATIONS IN THE CORONA VOLTMETER}

The corona voltmeter described by Whitehead and Isshiki (3) was used in these experiments. To obtain more convenient control of atmospheric conditions within the instrument and more accurate measurements of these conditions, the instrument has been modified by adding an extension to the bottom of the outer steel casing in the form of a chamber within which the control and measurement devices

${ }^{2}$ It should be noted that positive corona forms at lower voltages than negative for large rods at atmospheric pressure. For smaller rods and different pressures, negative corona may form at lower voltages. 
are located. Figure 1 shows a cross section of this chamber or subbase, while Figure 2 shows a cross section of the corona voltmeter with the subbase attached. The subbase is formed of a short piece of 16 -inch steel pipe $A$ with a flange at the top by which it is bolted to the bottom of the original instrument and another flange at the bottom for securing it to a cast-iron base $B$ carried on three legs made of $1 \frac{1}{2}$-inch iron pipe. In the center of the base is a cast-iron vessel $C$ with a central column $D$ through which passes a shaft carrying a fan for circulating the air. This fan is belt-driven by a motor not mechanically supported by the corona voltmeter. This precaution

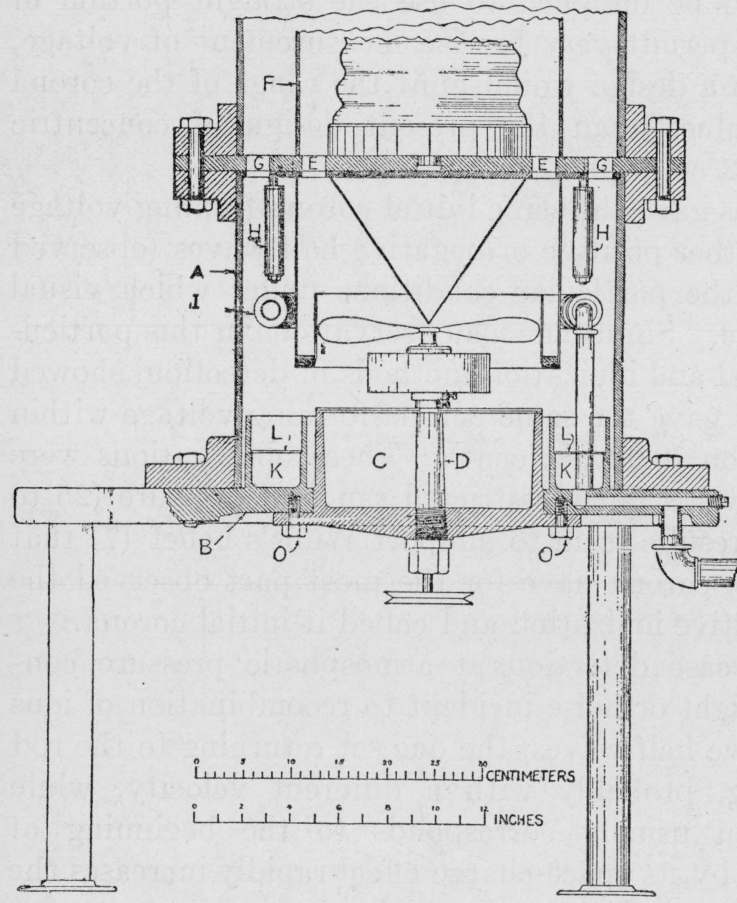

Fig. 1.-Subbase of corona voltmeter avoids the transmission of the hum of the motor to the telephone transmitter. The air is driven upward through a row of holes $E$ around the base of the pedestal insulator, is guided into the grounded electrode cylinder by means of a micanite tube $F$, and after flowing along the corona rod returns down the walls of the outer steel cylinder and reenters the base section through the holes $G$. Here it flows over a set of resistors $H$, which may be used to heat it, then over a cooling coil $I$, through which cold water may be circulated, and then reenters the fan. Either the heating coil or the cooling coil may be used, as conditions require.

The iron vessel $C$, which may be called the desiccator, is designed to contain five glass beakers for holding calcium chloride. A shutter arrangement covers the top of the desiccator, and by turning an external handle (not shown) the air may be allowed to pass over the drying agent, or be kept from it, as desired. This may be done while the corona voltmeter is in operation. The degree of opening of the shutter is indicated by a pointer and scale. 
When it becomes necessary to renew the calcium chloride in the desiccator $C$, the desiccator is lowered, after removing the holding screws $O$. Three guide rods, which are inserted in place of the first three screws to be removed, make it possible to lower the desiccator easily, while keeping it parallel to its original position, and to replace it in similar fashion.

When it was desired to humidify the air, water in measured quantities was evaporated in a small electrically heated metal cup inserted through a handhole.

The equipment provided by the new base section thus makes it possible to circulate the air, to heat or cool it, and -to dry it. A small motor-driven compressor was used to vary the air pressure in the corona voltmeter above or below atmospheric pressure. On the delivery side it was connected to a pressure tank fitted with a stopcock at the bottom to draw off condensed moisture. On the suction side it was connected to a tank to steady the flow of air from the corona voltmeter to the pump.

In order to dry the air for the corona voltmeter it was passed through a drying tube filled with lump anhydrous $\mathrm{CaCl}_{2}$. It could be by-passed around the drier tube, when desired, by proper manipulation of valves. The pipe line to the corona voltmeter passed near the manometer. At this position a needle valve $N$ (fig. 3 ) was located in the pipe line for coarse control of pressure by the manometer observer, who also had for fine adjustment of pressure a small needle valve $n$, used as vent cock. This valve was connected by heavy rubber tubing on the corona voltmeter side of the large needle valve.

The importance of having dust-free air was soon recognized, and, in addition to

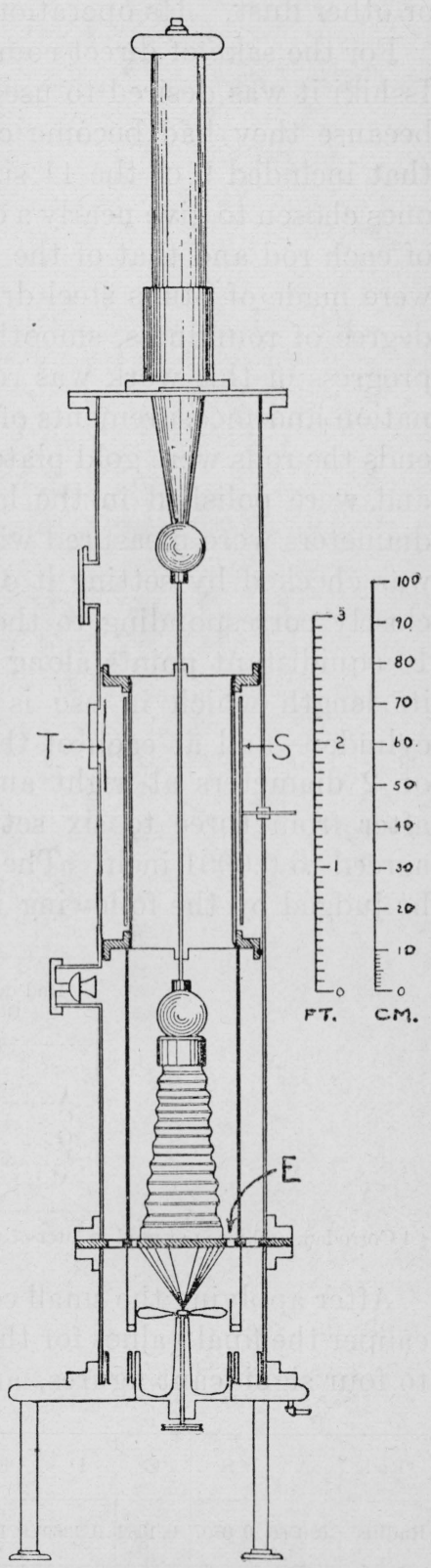

Fig. 2.-Corona voltmeter with subbase the air strainers provided in the drying tube, another air strainer was provided in the compressed-air pipe line near the corona voltmeter. 
This strainer consisted of a 2-inch tube 18 inches long, filled with absorbent cotton to remove any traces of oil, calcium chloride dust, or other dust. Its operation proved quite satisfactory.

For the sake of direct comparison with the work of Whitehead and Isshiki it was desired to use the same rods, but this was not feasible, because they had become corroded. A series of 13 rods was used that included 9 of the 11 sizes of Whitehead and Isshiki and 4 new ones chosen to give nearly a constant ratio of 1.2 between the diameter of each rod and that of the next smaller one of the series. The rods were made of Stubs steel drill rods carefully lapped to improve their degree of roundness, smoothness, and uniformity of diameter. The progress of this work was repeatedly checked by microscopic examination and measurements of the diameter. After attaching the brass ends the rods were gold plated to make them less subject to corrosion, and were polished in the lathe with chamois skin. After this the diameters were measured with an accurate micrometer caliper, which ${ }^{*}$ was checked by setting it on Johansson gauge blocks of thicknesses closely corresponding to the diameters. Each rod was calipered at 10 equidistant points along its working length - that is, that part of its length which in use is surrounded by the grounded electrode cylinder - and at each of the 10 points the measurement was made on 2 diameters at right angles. Each recorded value was entered after from three to six settings of the micrometer, which usually agreed to $0.0001 \mathrm{inch}$. The degree of roundness and uniformity may be judged by the following illustrative data:

\begin{tabular}{|c|c|}
\hline $\begin{array}{c}\text { Rod designa- } \\
\text { tion }\end{array}$ & $\begin{array}{c}\text { Range of observed } \\
\text { values of diameter }\end{array}$ \\
\hline & $\begin{array}{c}\text { Inches } \\
\text { A }\end{array}$ \\
\hline $\mathrm{C}$ & 0.0446 to 0.0450 \\
$\mathrm{G}$ & .0849 to $.0851_{5}$ \\
$\mathrm{~J}$ & .1797 to .1800 \\
$\mathrm{M} 13131$ to .3133 \\
.4998 to .5001 \\
\hline
\end{tabular}

1 Corroded, and was not used in later observations.

After applying the small corrections for the errors of the micrometer caliper the final values for the radii of the rods in centimeters, rounded to four significant figures, are as follows:

\begin{tabular}{c|c|c|c|c|c|c|c|c|c|c|c|c|c}
\hline Rod & $\mathrm{A}$ & $\mathrm{B}$ & $\mathrm{C}$ & $\mathrm{D}$ & $\mathrm{E}$ & $\mathrm{F}$ & $\mathrm{G}$ & $\mathrm{H}$ & $\mathrm{I}$ & $\mathrm{J}$ & $\mathrm{K}$ & $\mathrm{L}$ & $\mathrm{M}$ \\
\hline Radius & 0.0566 & 0.0797 & 0.1082 & 0.1369 & 0.1607 & 0.1961 & 0.2285 & 0.2765 & 0.3176 & 0.3976 & 0.4761 & 0.5548 & 0.6350 \\
\hline
\end{tabular}

It is believed that these figures are correct to 1 in the fourth decimal place. 
The inside diameter of the grounded electrode cylinder was measured at eight points along its axis and on four diameters $45^{\circ}$ apart at each point. The 32 values obtained ranged from 9.699 to 9.764 inches, the average being 9.727 inches. The correction to the micrometer used was -0.0006 inch. The value of the radius of the cylinder has, therefore, been taken as 4.863 inches $(12.352 \mathrm{~cm})$.

The cylinder was centered with respect to the axis of the rod of largest diameter, as well as could be done. It is estimated that the error of centering is probably not over $0.02 \mathrm{inch}$. The operation of centering is quite difficult, partly on account of the holes in the cylinder, which interfere with the use of the micrometer, and also because the cylinder is slightly elliptical.

It should be borne in mind that the grounded electrode cylinder in this corona voltmeter does not have the continuous surface which is implied in the simple theory of the instrument, but is perforated with about 2,900 holes $9.5 \mathrm{~mm}(0.375 \mathrm{inch})$ in diameter. In previous work with this corona voltmeter the effect of these holes upon the gradient at the surface of the corona rod has been tacitly assumed to be negligible. Because no formula was known to us by which an exact allowance for the effect of the holes could be made, we also have made no allowance for this effect. Dr. C. Snow ${ }^{3}$ has derived formulas for the analogous case of a cylinder having $n$ narrow, equally-spaced slits of equal width which are parallel to an element of the cylinder. From his work we estimate that the gradient at the surface of the largest rod we used is lower than the theoretical value for a cylinder with unbroken surface by about 0.14 per cent. In the construction of future instruments of this sort for research work when corona must be detected by the galvanometer method it would be advantageous either to perforate the cylinder with a sufficient number of considerably smaller holes; or better, to cut a large number. of equally spaced narrow slits in the cylinder, parallel to an element of its surface. This latter construction would make it possible to compute the correction to the gradient by Snow's formula.

\section{TEMPERATURE MEASUREMENT}

Aside from the introduction of the micanite tube for guiding the air current, the upper or original part of the corona voltmeter was left substantially unchanged, save in the use of thermocouples to obtain an accurate measurement of the temperature of the air near the corona rod. A set of six thermocouples was installed with the six "hot junctions" just above the holes $E$ (fig. 2) and the "cold junctions" between the outer casing and the inner cylinder (that is, near the upper end of the mercurial thermometer $T$ ). Thus, one set of junctions is

\footnotetext{
${ }^{3}$ Electric Field of a Charged Wire and a Slotted Cylindrical Conductor, Chester Snow, B. S. Sci. Paper No. 542, 1926.
} 
in the air stream as it leaves the fan and is about to flow up through the "working space" around the corona rod, while the other set is in the air stream which has just left this space. The two terminals of this battery of thermocouples extend to the potentiometer. The potential difference between these terminals is a measure of the temperature difference of the air stream at the two points. By running the fan the temperature difference could be kept down to a small value (usually less than $0.2^{\circ} \mathrm{C}$. and often less than $0.1^{\circ} \mathrm{C}$.), thus insuring a very uniform temperature of the air along the corona rod.

Another set of six thermocouples is installed with three of the hot junctions just above the holes $E$ (fig. 2) in the air entering the working space, and the other three in the air which has just left the top of the working space. The six cold junctions are brought outside the corona voltmeter and are located in melting ice in a thermos bottle. (See fig. 3.) These thermocouples were accurately checked by the heat division of the Bureau of Standards before they were installed in the corona voltmeter.

Another set of thermocouples with cold junctions in melting ice was distributed along the length of the tube and scale of the mercurial manometer, and their electromotive force gave an accurate measure of the average temperature of the mercury in the column.

The potentiometer used with the thermocouples was made up for the purpose by reconnecting a Wolff precision rheostat and mounting it and all necessary keys, galvanometers, etc., on a baseboard. It is designed so that thermal emf's resulting from the friction of the dial brushes are excluded from the measurement circuit. Its reading is approximately in ${ }^{\circ} \mathrm{C}$., with a range of $0^{\circ}$ to $50^{\circ} \mathrm{C}$., which may on occasion be doubled by doubling the auxiliary current and using two standard cells in series where one is normally used. If the relation between emf and temperature for the copper-constantan thermocouples used were strictly linear with temperature, the potentiometer could readily have been designed to read very exactly in ${ }^{\circ} \mathrm{C}$. Since there are small second and third degree terms, the potentiometer has been designed on the basis of the slope of the temperature-thermal emf curve at average room temperature of $25^{\circ} \mathrm{C}$. Thus, one can tell the approximate temperature by a glance at the dials, while to reduce the readings to exact values a curve of corrections is used.

To give maximum convenience and speed in operation, the potentiometer was designed to use a Weston pivoted moving-coil galvanometer of short period and to operate on either the null or the deflection principles, as may be necessary - that is, if the operator is not otherwise engaged, he can read the temperature to the nearest $0.01^{\circ} \mathrm{C}$., by successive adjustment of the dials until the galvanometer deflection is zero. But if he is so busy that he can not keep the dials adjusted for this condition, he can leave them alone and note 
the number of divisions deflection to right or left of zero. The potentiometer circuits are so compensated and related to the sensitivity and resistance of the galvanometer and the resistance of the thermocouple circuits that each division of galvanometer deflection corresponds to $0.1^{\circ} \mathrm{C}$, and since the position of the pointer can be read to 0.1 division, it is possible to detect a change of $0.01^{\circ} \mathrm{C}$. This deflection feature is of especial convenience when a definite temperature is to be maintained in the corona voltmeter. In this case, the potentiometer dials are set to the e. m. f. corresponding to this temperature and left so. Then the operator can tell, from the position of the pointer, whether the air is above or below the desired temperature and how much, and he can apply heating or cooling as may be necessary. For example, if it is agreed that a deviation of, say, $0.1^{\circ} \mathrm{C}$. either way (corresponding to 0.03 per cent in corona voltage) may be ignored, he need only see that the pointer does not get more than one division away from zero.

\section{PRESSURE MEASUREMENT}

The manometer $U$, shown in Figure 3, is of the open-tube type, arranged to read the pressure of the air in the cylinder above or below atmospheric pressure. The left-hand branch of the $U$ tube is connected to the corona voltmeter, and the right-hand branch is open to the atmosphere. When the air in the voltmeter is in equilibrium with the atmosphere the mercury half fills each branch, and its level coincides with the central zeros of the two scales. These scales are $3 \mathrm{~m}$ long, divided in millimeters. When the pressure of the air in the voltmeter is greater than atmospheric pressure the mercury rises in the right-hand branch and sinks in the left, and the sum of the readings of the two scales gives the pressure above that of the atmosphere. Readings on the right-hand scale below the zero and those on the left-hand scale above the zero are considered negative, and with this convention no error will be introduced by failure to adjust the zeros of the scales initially to coincide with the mercury level.

When the pressure of the air in the voltmeter is below atmospheric pressure the mercury rises in the left-hand tube and sinks in the right, and the sum of the two (negative) scale readings gives the amount by which the pressure in the voltmeter is lower than atmospheric pressure. The range of the manometer is from a vacuum to $300 \mathrm{~cm}$ above atmospheric pressure. Our barometer was checked against the standard barometer of the Bureau of Standards, and the correction was found to be less than $0.1 \mathrm{~mm}$. The readings of the manometer and barometer were reduced to $0^{\circ} \mathrm{C}$. and latitude $45^{\circ}$, using data from the Smithsonian Physical Tables. 


\section{DETERMINATION OF HUMIDITY}

The humidity of the air was determined with a dew-point hygrometer (fig. 4), which may be used over a wide range of pressure. Necessary cooling was obtained through the Joule-Thomson effect by expanding compressed carbon dioxide in copper tubing within the copper shell. The hygrometer was designed with its parts concentric, so that uniform heat flow could be expected from the observed central spot of the cooling coil. The observed field of view consisted of two semicircles-one, $A$, the dew-forming surface of chromium-plated polished silver; and the other, $B$, a comparison field of chromiumplated polished metal, maintained at a temperature slightly above that of the other surface. The two observed mirror surfaces were illuminated by four flash-light lamps provided with screens of green glass, $S$, to intercept heat radiation, while specular reflection to the eye from the mirror surfaces was that of a black velvet surface properly located in front of the mirror surfaces. (Black velvet reflects about 2 per cent of incident light.) The first formation of dew or frost on the cooled mirror surface, $A$, is readily visible because of diffuse reflection by the tiny particles of moisture which show up quickly in contrast with the warmer mirror surface, $B$, which reflects only a portion of the light diffusely reflected from the black velvet.

The design finally adopted employed a single small thermocouple soldered on the back of the cooled mirror disk at its center. The cold junction was kept in an ice bath. The resistance of the thermocouple circuit was adjusted for convenience in reading temperatures directly on the Paul microvoltmeter. The combination was calibrated, using certified mercurial thermometers.

A small heating coil on a copper cylinder mounted concentric with the mirror disk facilitated removal of dew from the surface at high humidities and thereby shortened the time necessary to take dewpoint readings.

\section{DETECTION OF CORONA FORMATION}

Because of its inferior sensitivity to higher audible frequencies, the ordinary telephone transmitter or microphone shown in Figure 2 was replaced by a condenser microphone. Aural observation of corona consisted in picking up the faint corona sound by means of this condenser microphone and, after vacuum-tube amplification, listening for the amplified sound with head phones. In order to use the condenser microphone $T$ (fig. 4 ), devised by A. Hund, a special housing, $A$, (fig. 3 ), was made to fit one of the flanges on the corona voltmeter. The leads to this transmitter entered the corona voltmeter through commercial gas-engine spark plugs. It was necessary to shield this transmitter and its amplifier electrostatically. In Figure 3 the resistance-coupled amplifier and storage cells used inits 
B. S. Journal of Research, RP21

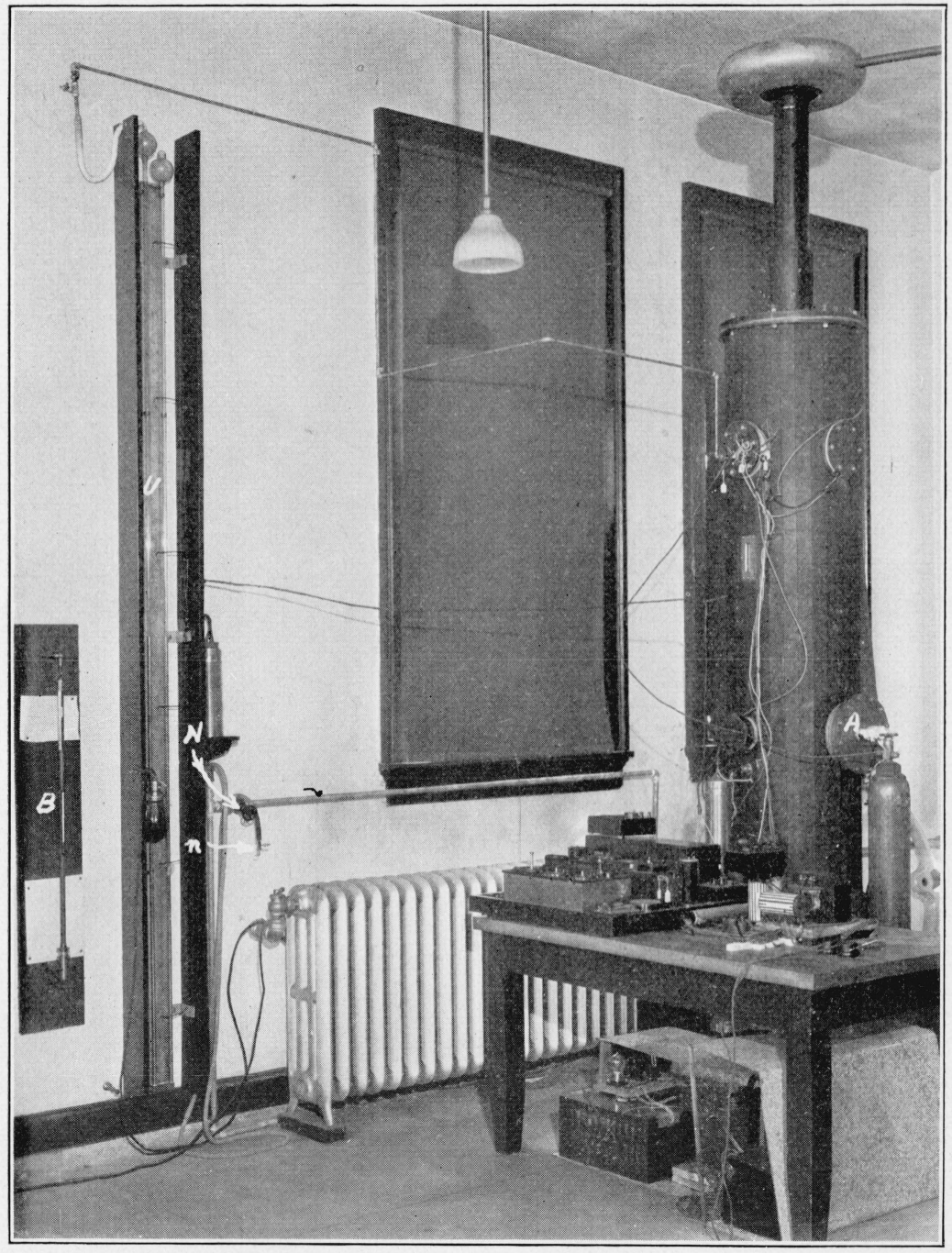

FIG. 3.-Corona voltmeter with manometer, barometer, thermocouple potentiometer, and other accessory apparatus 
B. S. Journal of Research, RP21

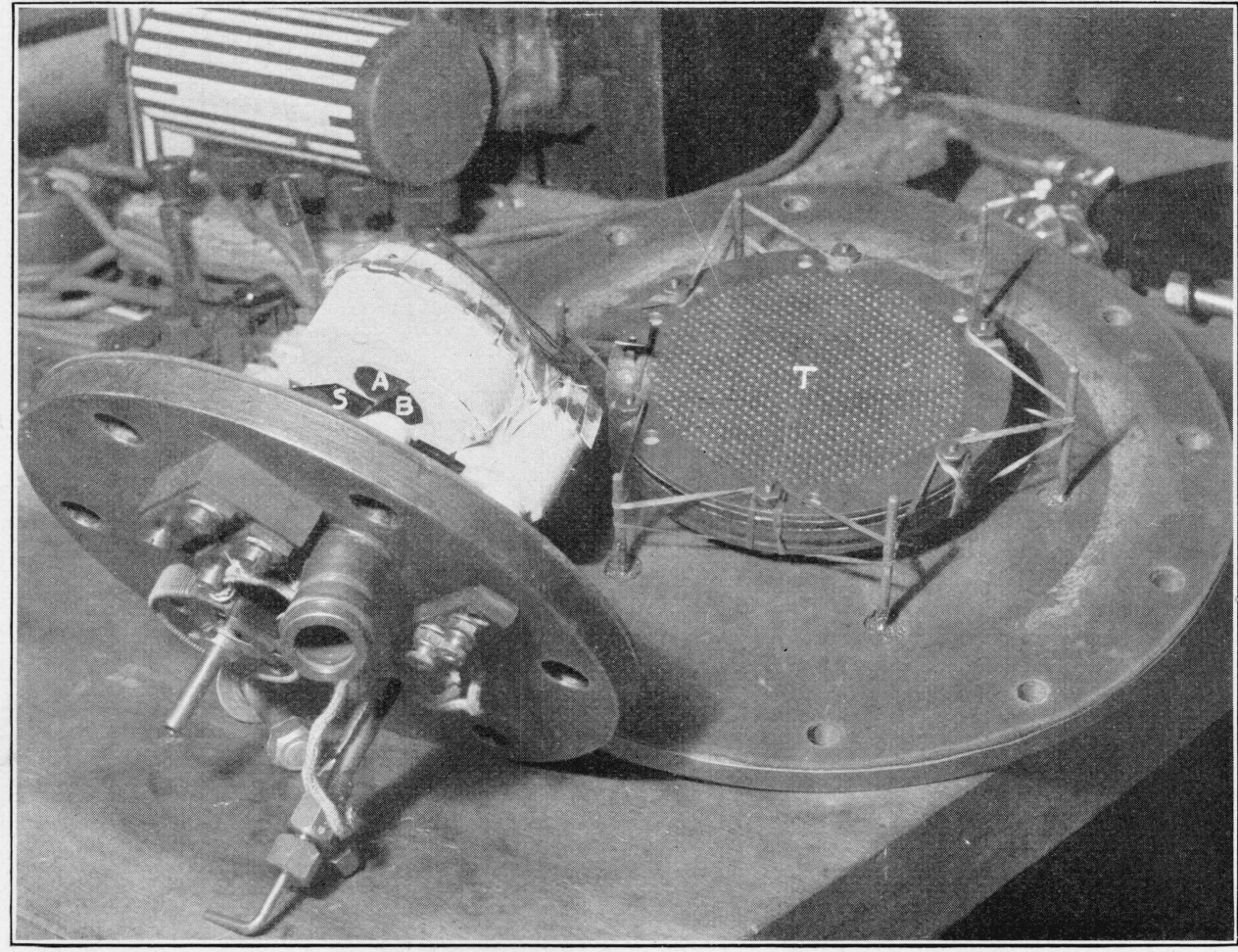

FIG. 4.-Dew-point hygrometer (left) and condenser microphone (right) 
operation are shown. In order to eliminate leakage difficulties at high humidities, it was found highly desirable to heat the grid-lead spark plug and the condenser transmitter by a heating coil attached to the lighting circuit.

Ionization detection of corona formation was accomplished as follows: A 100-volt battery (fig. 5) in series with a galvanometer was connected between the outer (perforated) concentric cylinder (fig. 2) and a third insulated cylinder surrounding it. Passage of ions through the perforated cylinder to the insulated cylinder was indicated by a deflection of the galvanometer. A Leeds \& Northrup "portable lamp and scale" galvanometer of 325 ohms resistance and 27 megohms sensitivity was used. In the case of very high humidity, leakage across the insulation of the insulated cylinder of the corona voltmeter became troublesome. To reduce the initial

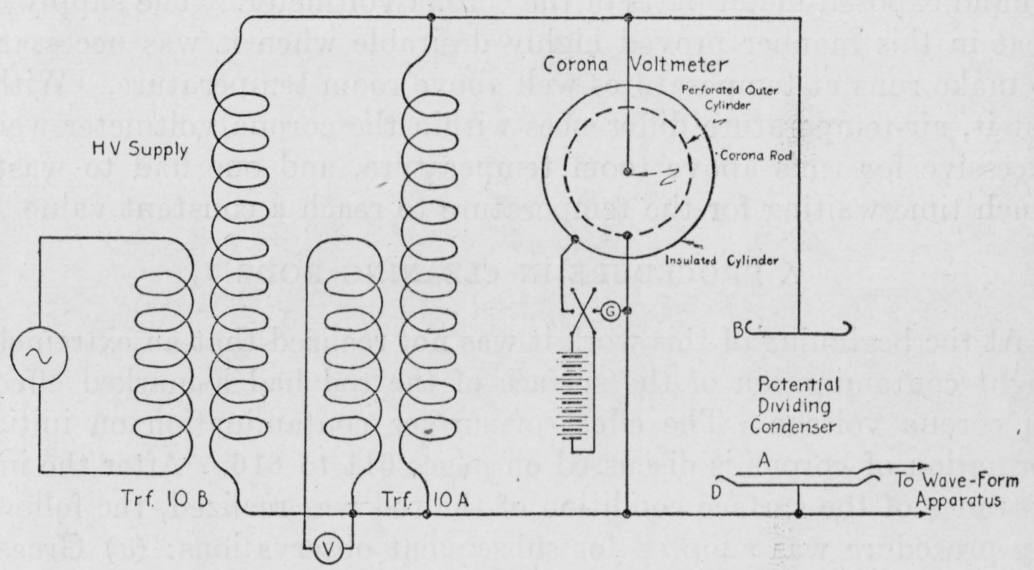

Fig. 5.-Diagrammatic plan of connections of corona voltmeter, supply transformer, voltage transformer (10A) and potential-dividing condenser

deflection produced by this leakage current to zero, recourse was had to the usual method of shunting the galvanometer with a very high resistance circuit containing a small e. $\mathrm{m}$. f. from an auxiliary dry cell. This procedure produced a calculated decrease in sensitivity of 10 per cent. It was customary to maintain the insulated cylinder $\mathrm{S}$ (fig. 2) at 100 volts negative, except in the case of low pressures and small rods, for which case $\mathrm{S}$ was maintained at 100 volts positive.

For one set of observations it seemed desirable to check visual detection of corona against the aural and ionization methods. To do this, a small glass window capable of withstanding the pressures used in the corona voltmeter was made for one of the handhole blank flanges. By means of a mirror cemented to the upper corona-rod fitting so as not to disturb the function of the rod, it was possible to observe the corona glow along the entire length of the rod. The $111896^{\circ}-28-3$ 
stroboscope disk placed in front of the window was cut away for $180^{\circ}$, so that, when mounted on a two-pole synchronous motor, reversal of the d. c. field permitted observation of the corona glow for either half wave. Because of the very faint luminosity resulting from initial corona, it was necessary to darken the room and keep extraneous light from reaching the observer by the use of black cloth.

\section{TEMPERATURE CONTROL}

In order to reduce the heat transmission through the walls of the corona voltmeter, it was covered with a layer of cork board 1.5 inches thick. This greatly facilitated the maintenance of a definite air temperature within, especially when this differed considerably from that of the air in the room.

One may also see in Figure 3 that heating coils have been wound around exposed metal parts of the corona voltmeter. The supply of heat in this manner proved highly desirable when it was necessary to make runs at temperatures well above room temperature. Without it, air-temperature differences within the corona voltmeter were excessive for runs above room temperature, and one had to waste much time waiting for the temperature to reach a constant value.

\section{PROCEDURE IN CLEANING RODS}

At the beginning of this work it was not realized that an extremely slight contamination of the surface of the rod had a marked effect on corona voltage. The effect of surface contamination on initial formation of corona is discussed on pages 611 to 616 . After the importance of the surface condition of the rod was realized, the following procedure was adopted for subsequent observations: (a) Grease and oil were removed by wiping the corona rod with a clean rag; $(b)$ the rod was washed with high-grade benzene; $(c)$ it was polished with crocus paper; $(d)$ it was washed with redistilled ethyl alcohol; and $(e)$ it was wiped with specially cleaned chamois skin from which tanning residues had been removed by washing with soap, distilled water, and washing and drying with redistilled alcohol. An attempt was made to keep all dust off the wiping surface of the chamois. The chamois itself was touched only by hands which had been thoroughly washed with soap and water, and an attempt was made to handle the chamois skin without touching the wiping surface with the hands. The corona-forming surface of the "cleaned" rods was not touched between the cleaning process and use in the corona voltmeter; $(f)$ at times the rod was again sprayed with redistilled alcohol and allowed to dry, but this additional treatment did not seem to alter the behavior of the rod treated as in operations $(a)$ to $(e)$. 


\section{PRODUCTION AND MEASUREMENT OF VOLTAGE}

\section{SOURCES OF VOLTAGE}

The General Electric Co. motor-generator set used in this investig ation was of a recent type specially designed to give as nearly as possible a sine wave form of terminal voltage under all conditions of load. It consists of two three-phase machines driven by a d. c. motor, with means for shifting the stator of one of the alternators through 360 electrical degrees. To facilitate the work of tracing the wave form, an accurately divided are was put on, with a vernier reading to 0.1 electrical degree. Preliminary trials with the oscillograph showed that the voltage wave form of these machines is very nearly sinusoidal, even for the very unfavorable case of full rated volt-amperes at zero power factor, current leading. To facilitate the work of tracing the wave form accurately, this set of machines is located in the same room with the corona voltmeter and the apparatus for obtaining the wave form.

Considerable difficulty was experienced in obtaining the desired constancy of voltage and speed of the above-mentioned machine. Several improvements of both mechanical and electrical natures were made before measurements were undertaken. The desired constancy of speed was finally obtained by running another motorgenerator set with very large moment of inertia from a storage battery to supply one of the generators of the three-phase set run as a synchronous motor. Using this arrangement, the voltage at the motor terminals was found not to fluctuate by more than about 0.01 per cent. The other three-phase generator was used to supply the transformers connected to the corona voltmeter and high-voltage wave-form apparatus.

\section{MEASUREMENT OF EFFECTIVE VOLTAGE}

The methods used by previous investigators in measuring the effective value of high alternating voltages have usually involved the assumption that the secondary (high) voltage of a transformer is equal to the product of the primary (low) voltage multiplied by the ratio of secondary to primary turns. The use of the so-called voltmeter coil or tertiary coil is a variant and has some comparative advantages and shortcomings. However, both of these methods neglect the rise of voltage at the secondary terminals due to the capacitance of the sections of the high-voltage winding with respect to each other and to the grounded core and case. Furthermore, the corona voltmeter is a condenser, and the leading current taken by it adds its effect. The result is an error whose direction is known, but whose magnitude can only be guessed at. In the average case, as far as previous work is concerned, it probably amounts to several per cent at 60 cycles and may become excessive at high frequencies. 
Investigations at this bureau over a period of years have shown that the step-down ratio and phase angle of certain transformers are not sensibly affected by the capacitance currents between their highvoltage windings and from them to the grounded parts. The particular transformer used in this investigation has high-voltage ranges of 25,50 , and $100 \mathrm{kv}$. Its ratio and phase angle have been repeatedly checked by the potentiometer method up to $30 \mathrm{kv}$ with natural cooling of a shielded resistor (8), and up to $50 \mathrm{kv}$ with forced ventilation; that is, it has been tested with its high-voltage coils in parallel, and also two parallel, two series up to rated voltage; also in series connection up to $50 \mathrm{kv}$. By means of supplementary methods, values of ratio and phase angle have been obtained up to the maximum voltage of $100 \mathrm{kv}$ with an accuracy which is estimated to be only slightly inferior to that obtained by the absolute (potentiometer) method. The extrapolation of ratio and phase-angle curves for these $100 \mathrm{kv}$ transformers over the range 50 to $100 \mathrm{kv}$ has been carried out on the following principles: First, assume that both the primary and the secondary windings were of such low voltage that capacitance-current effects would vanish. In this case the ratios for the three cases- $(a)$ primary coils all in parallel; $(b)$ two in series, two parallel; $(c)$ all four in series-will be precisely in the ratio $1: 2: 4$, since the four coils are very accurately alike both electrically and magnetically. Also, if the ratio factor (quotient of actual ratio divided by nominal ratio) be plotted as a function of secondary voltage at any given frequency and given load (expressed in voit-amperes at a stated power factor), its deviation from a constant value will be due solely to the varying magnetic permeability and core losses with flux density; and furthermore, the ratio-factor curves and the phase-angle curves for the three methods of connecting the secondary coils will coincide. In such a case, if means were at hand for accurately determining the ratio and phase angle of the transformer on only one range (for example, with the four sections of the primary winding in parallel), the ratios of the other two ranges could be taken as being accurately twice and four times the ratio as measured with the coils in parallel.

Now, let the four primary coils be assumed to be wound with many turns for high voltage, so that capacitance currents of appreciable magnitude flow in a rather complicated manner from turn to turn, layer to layer, section to section, and between each one of these many elements and the grounded core and case. Although the magnitude and the distribution of these currents are unknown, it can be seen that the various elements of electrostatic capacitance with the resistances and the self and mutual inductances of the parts of the winding constitute a network, and that the effect of the capacitance currents on the secondary voltage will be a constant relative amount, 
regardless of the magnitude of the voltage; that is, if the capacitance-current effect is an increase of 0.2 per cent in voltage with a secondary voltage of $50 \mathrm{kv}$, it will still be 0.2 per cent at $100 \mathrm{kv}$. This applies to the case of a given manner of connecting the four sections of the secondary winding and neglects second-order terms such as may enter because of the variation of the dielectric constants of the insulating materials as the gradient varies. Since theory and experiment show that the entire effect of capacitance currents on ratio and phase angle is small, these second-order terms may be neglected. Consequently, if the relative effect of the capacitance currents can be determined at one voltage, it may be taken to apply

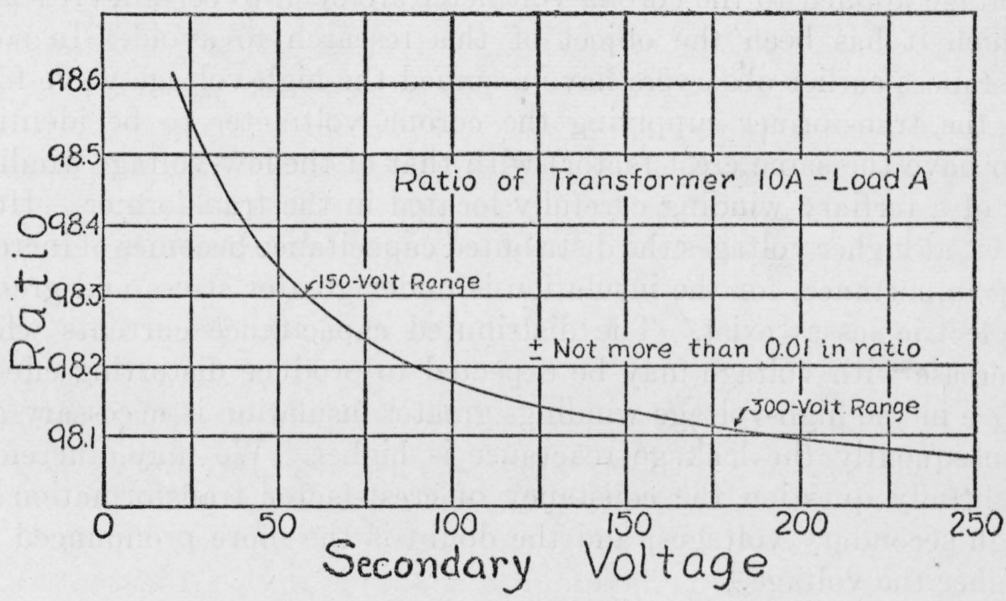

FIG. 6.-Ratio of voltage transformer $10 \mathrm{~A}$ as a function of secondary voltage

The change at 150 volts is caused by the change in secondary load when the voltmeter range is altered

to higher voltages for which the means at hand do not permit a direct determination.

In Figure 6 the ratio of transformer $10 \mathrm{~A}$ is plotted for the case of the particular secondary burden resulting from auxiliary apparatus used in conjunction with the corona voltmeter. The sudden shift of this curve at 150 volts is the result of changing from 150 to 300 volt ranges of connected instruments.

The secondary voltage of this step-down transformer was measured with a precision electrodynamic voltmeter, $V$ (fig. 5), of the reflecting type. It embodies several novel features, including a device for obviating the usual uncertainty caused by drift of zero, compensation for the effects of varying room temperature, and an optical double-lever arrangement which gives such sensitivity of reading that a change in voltage of 1 in 100,000 can be detected. It is checked from time to time during the progress of a set of observations, by reference to a standard cell and resistances of known value. This voltmeter 
will be completely described in a forthcoming Bureau of Standards publication by $\mathrm{F}$. K. Harris. It may be seen that with such a degree of refinement in the measurement of the secondary voltage the determining factor is the accuracy with which the step-down ratio of the transformer is known.

\section{DETERMINATION OF WAVE FORM AND CREST FACTOR}

In obtaining crest voltage the determination of crest factor is of no less importance than the measurement of effective voltage and the determination of ratio of transformation just mentioned.

Previous methods of determining the wave form of the alternating voltage applied to the corona voltmeter are open to certain criticisms which it has been the object of this research to avoid. In some instances earlier observers have assumed the high-voltage wave form of the transformer supplying the corona voltmeter to be identical (to have the same crest factor) with that of the low-voltage winding, or of a tertiary winding carefully located in the transformer. However, at higher voltages the distributed capacitance becomes of increasing importance, for the insulation is under greater stress and greater dielectric losses exist. The distributed capacitance currents which increase with voltage may be expected to produce distorting effects, since in the high-voltage windings greater insulation is necessary and consequently the leakage reactance is higher. We may, therefore, rightfully question the constancy of crest-factor transformation for high secondary voltages, and the doubt is the more pronounced the higher the voltage.

Recognizing the necessity of experimental proof of all assumptions regarding constancy of wave-form transformation, Whitehead and Isshiki (3) carried on an extended investigation with the synchronous commutator and with kenotrons in conjunction with a guarded air condenser on the high-voltage side of their transformer. By careful work their two methods were shown to be in good agreement. The method used is this work differs from the above methods in that it is a null electrostatic point-by-point method and has the essential advantages of null methods over deflection methods. The condenser $B A D$ (fig. 5) is a voltage-dividing condenser which is shown connected to the secondary of transformer $10 B$. By means of this condenser a small portion, say 100 volts, of the high alternating voltage $(8,000$ to 95,000 volts) is obtained. It is evident that the wave form of this portion is identical with that of the total high voltage, because there is nothing in the voltage-dividing arrangement to introduce distortion.

A point-by-point method of ootaining wave form consists of balancing the instantaneous voltage for selected points against an accurately known continuous voltage. Using these carefully deter- 
mined values and their corresponding angular settings, we may plot the wave form, using as many points as we care to observe; or, if we make our observations at the proper angular interval, we may calculate the crest factor directly from the settings without plotting the entire wave form. One should realize that the above balance of instantaneous values refers to an average instantaneous value of the same relative location in many successive cycles, and that for the duration of balance the effective voltage must be kept constant, and that the effective voltage must be the same for all balances in any one wave form. This method of high-voltage crest-factor deter-

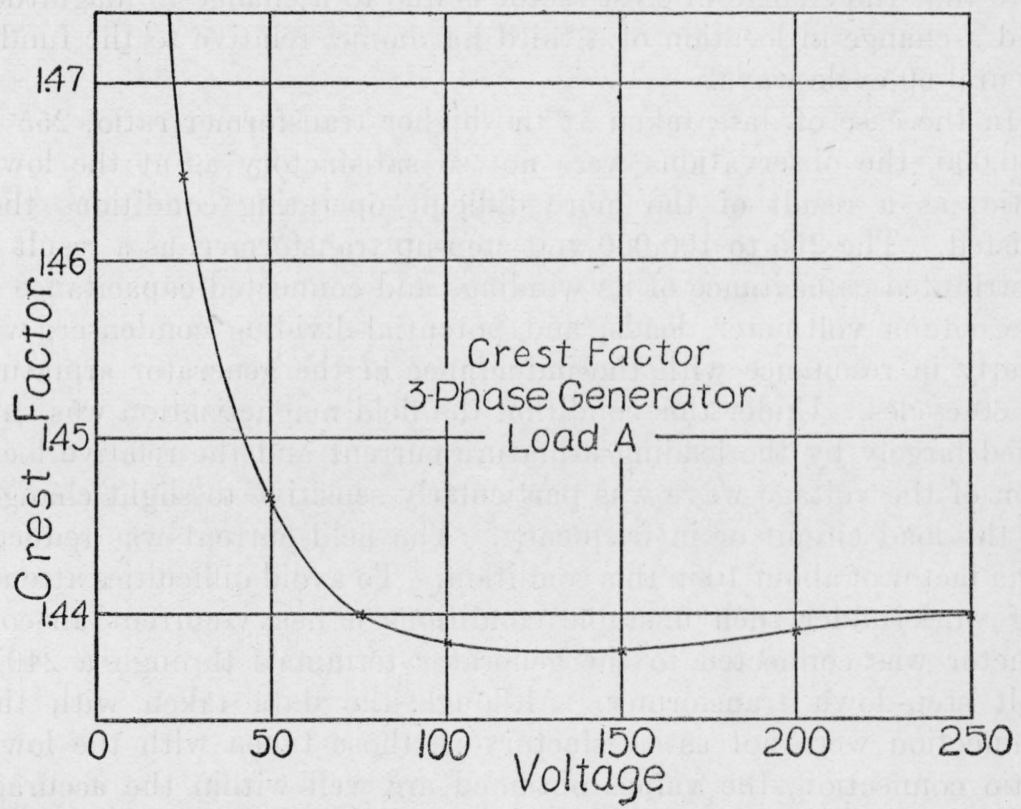

Fig. 7.-Crest factor of high secondary voltage applied to the corona voltmeter, as a function of the secondary voltage of the step-down transformer

mination will be described in a forthcoming Bureau of Standards publication.

It is important to mention the fact that the secondary voltage wave form is dependent upon the nature and magnitude of the load on the machine. For this reason, in order to simplify computations and experimental work, it was decided to use only two transformer connections, namely, 255 to 100,000 and 255 to 25,000 , to connect the supply-machine windings always in series, and to use only two instrument ranges, of 0 to 150 and 0 to 300 volts, respectively. Wave-form data were therefore taken for these connections for different voltages and crest factors and are shown plotted in the case of the 255 to 25,000 volt connection in Figure 7 . The curves as plotted are crest factor of the high voltage against voltage as measured 
by the precision voltmeter $V$ (fig. 5 ) on the low-voltage side of transformer $10 \mathrm{~A}$. The $50,100,150,200$, and 250 volt points were first taken and plotted. Since the 50 -volt point appeared inconsistent with the rest, repeat runs on the 50 -volt point were made and found to check each other closely (the average deviation of four runs from the mean for this point being 0.03 per cent). The 25 and 75 volt points were then taken and found to fall on the curve through the 50 -volt point.

From this curve, through observed points, the values of intermediate crest factors were taken. Harmonic analysis seems to indicate that the change in crest factor is due to a change in magnitude and a change in location of a third harmonic, relative to the fundamental 60-cycle wave.

In the case of data taken at the higher transformer ratio, 255 to 100,000 , the observations were not so satisfactory as at the lower ratio, as a result of the more difficult operating conditions that existed. The 255 to 100,000 volt step-up transformer, as a result of distributed capacitance of its windings and connected capacitance of the corona voltmeter, leads, and potential-dividing condenser, was nearly in resonance with the inductance of the generator armature at 60 cycles. Under this condition the field magnetization was supplied largely by the leading armature current and the relative location of the voltage wave was particularly sensitive to slight changes in the load circuit or in frequency. The field current was reduced by a factor of about 10 in this condition. To avoid difficulties attending work under such unstable conditions a heavy-current air-core reactor was connected to the generator terminals through a 240:6 volt step-down transformer. Although the data taken with this connection were not as satisfactory as those taken with the lower ratio connection, the values obtained are well within the accuracy obtainable with the corona voltmeter. Since the same connections are used in each of the two cases-dry and humid conditions within the corona voltmeter - the relative effects of humidity are quite accurately obtained, while the absolute accuracy of the data is greater than the accuracy obtainable on repeating observations of initial corona formation.

\section{OBSERVATIONS ON THE CORONA VOLTMETER}

\section{DESCRIPTION OF OBSERVERS' DUTIES}

It was found expedient to use four observers in most of the runs. The first observer, who was provided with head phones for aural detection of corona, was also provided with a slide rheostat in the generator field circuit to permit very fine control of the supply voltage over the range of initial formation and cessation of corona, and he observed the effective value of the corona-forming voltage on the 
precision voltmeter previously described. In what we have designated as a "run" in this discussion the procedure was to take at least three consecutive readings of voltage for initial formation of corona while air pressure and air temperature were maintained at a constant value. If the individual values of voltage did not agree to within a few hundredths of 1 per cent, additional points were taken or the cause of disagreement was remedied and a new set of three voltage readings was taken and recorded. The average value from three or more consecutive readings taken in this way gives the formation voltage for corona for that particular run. Between corona runs the first observer calibrated his voltmeter and ascertained that the connected load corresponded to that previously used in determining crest factor. The second observer, who recorded observations of his own and those of the first observer, made the necessary major adjustments in supply voltage, maintained nearly constant frequency of the supply, checked aural observations of the first observer using the ionization-detection galvanometer, and as a safety precaution was in position to cut off the power supply in case of spark over. The third observer was primarily concerned in manipulating the air supply for the corona voltmeter. By constantly adjusting needle valves, he maintained the pressure constant within the corona voltmeter while other observations were being made. He also made dew-point observations. The fourth observer, who recorded his own readings and manometer readings for the third observer, also measured temperatures by means of the thermocouple potentiometer, observed the dew-point temperature and the barometric pressure, and assisted in maintaining desired temperatures within the corona voltmeter by regulating the heating current. For runs at atmospheric pressure a single observer replaced the third and fourth observers.

\section{SOURCES OF ERROR}

Transformer-ratio, primary-voltage, and secondary crest-factor measurements have been discussed. The frequency of the supply was kept constant to within about 1 part in 300 . Experiments show that a change in frequency of this magnitude will alter the crest factor by an amount which, though definitely observable, is less than we need consider. Observations of others indicate that such a slight change would not alter the corona-forming voltage appreciably, and our observations show that a change in frequency of this order does not affect the transformer ratio or the primary voltmeter readings. It is estimated that the over-all accuracy of our determination of crest voltage is better than 0.1 per cent.

The method of temperature measurement has been discussed. Potentiometric readings of the air-temperature difference indicate that, although our electrical sensitivity enabled us to observe readings 
corresponding to an accuracy of $\pm 0.01^{\circ} \mathrm{C}$., we do not know the actual air temperature or air-temperature difference with corresponding accuracy, especially if the corona-voltmeter temperature is somewhat above or below room temperature, for it is impossible to bring all parts of the corona voltmeter to a common temperature. On the average, temperature measurements are probably good to $0.1^{\circ} \mathrm{C}$. The circulating fan was extremely useful in reducing the temperature difference between the air in the top and bottom of the corona voltmeter. If allowed to stand overnight without fan circulation, the air-temperature difference would sometimes amount to more than $1.0^{\circ} \mathrm{C}$. Fan circulation would soon reduce this figure to $0.01^{\circ} \mathrm{C}$. Heating or cooling could not be uniformly applied to the air and to the corona-voltmeter parts, and one might suppose that, although the air was continually stirred, certain small volume elements leaving the heating coils would have temperatures much higher than the average. On the other hand, the corona-forming surface was well insulated electrically and, therefore, thermally from any artificially warmed metallic surfaces. It was also sufficiently removed from the heating units that the heated portions of air should be well mixed with other portions of the air before reaching it. Since the air flow is not restricted in the region near the corona rod, the air velocity there is low and the layer adjacent to the rod in which corona forms must have substantially the same temperature as the rod. Fortunately, a deviation in temperature of $0.1^{\circ} \mathrm{C}$. corresponds to a difference in corona voltage of but 0.03 per cent. It was customary not to take corona runs unless the air-temperature difference was low. In any event, air-temperature difference is recorded for each run, and those readings for which this difference seems excessive have been excluded from consideration. A discussion of desirable accuracy of temperature measurement is given in the appendix.

The principal source of error in the measurement of the pressure was the error in reading the manometer. Although the menisci varied in shape somewhat, the internal diameter of the manometer tubing was $1 \mathrm{~cm}$, so that the maximum error in pressure measurement by the manometer was probably less than $\pm 0.07 \mathrm{~cm}$. Therefore we may state that at the lowest observed pressures our accuracy of pressure measurement was better than \pm 1 per cent and increased linearly to better than \pm 0.1 per cent at atmospheric pressure. A discussion of the accuracy desired in pressure measurement is given in the appendix.

Dew-point observations could be made with an accuracy of about $\pm 0.2^{\circ} \mathrm{C}$., which seems amply sufficient for this work.

A discussion of the effect of errors in observed values of the radii of the corona rods and cylinder, and of air pressure and temperature, is included in the appendix. 


\section{COMPUTATIONS AND CHECKING}

The necessary small corrections were applied to the readings of the precision voltmeter. These corrections were in general less than 0.1 per cent.

The quantity of saturated water vapor in grams per cubic meter (9) was taken from a graph of that quantity as ordinates against temperature as abscissas, the temperatures used being those of the dewpoint hygrometer and the air within the corona voltmeter. Absolute humidity or ratio of water molecules to air molecules present is used as the basis of presentation of the effect of moisture, since the relative humidity basis of consideration of water vapor present apparently did not lead to better correlation of results. It was usually possible to detect errors in computation by plotting the deviation of a set of corona runs from some arbitrarily chosen smooth curve. If certain computed values did not fall reasonably close to a smooth curve plotted through most of the points, then these computations were rechecked. Since over 30 distinct computations were necessary for each run, and there were in the neighborhood of 500 runs, the need for guarding against errors in computation was particularly great.

\section{RESULTS}

\section{METHOD OF PRESENTING DATA}

The data were originally computed on the basis of the law of corona

$$
g=A \delta+B \sqrt{\delta / r}
$$

It was found convenient to check observations and computations in a simple way by using residuals obtained by subtracting a quantity

$$
g^{\prime} / \delta=30+9 / \sqrt{\delta r}
$$

from the observed values of $g / \delta$ obtained experimentally-that is, $A$ was arbitrarily chosen equal to $30 \mathrm{kv} / \mathrm{cm}$, and $B$ equal to 9 in equation (4). For experimental values of $\delta$ and $r$, values of $g^{\prime} / \delta$ were computed. The difference between these values of $g^{\prime} / \delta$ and the experimental values $g / \delta$ - that is, the residuals - was plotted against $1 / \sqrt{\delta r}$. This method of plotting provided a fairly powerful tool for checking purposes, as it is equivalent to plotting $g / \delta$ on a very open seale. If the plotted values of residuals against $1 / \sqrt{\delta r}$ did not lie fairly close to smooth curves, they were inspected for computation errors.

For any one rod, the above law (4) did not give a single straight line. Evidence of this fact may be observed in the original work of Whitehead with the corona voltmeter. In this work Whitehead also found that the data on all rods were approximately satisfied by two intersecting straight lines. More accurate measurements were pos- 
sible with the equipment at our disposal, and our results indicate that a smooth curve fits the observed data for a single rod somewhat better than a straight line when plotted on the above basis, and that the envelope of values for different rods appears to be a smooth curve rather than two straight lines. In a later paragraph an equation is presented which fits experimental data more closely than the above relation (4). However, it has been found expedient to show the effects of contamination of the surface of the rod and the presence of water vapor and other impurities in the air, using residuals determined as above with regard to equations (4) and (5).

The actual observations and computations are so numerous that they may not be presented in entirety. We have, therefore, so far as possible, presented our results in the form of graphs.

\section{EFFECT OF CONTAMINATION OF ROD SURFACE}

It is well known that dust on the corona-forming surface lowers the corona voltage. The following experience with the $\operatorname{rod} A$ shows that it is desirable to have entirely dust-free air: A run on $\operatorname{rod} A$ was found to give a value for full corona sound lower than the "dry-air" value of corona voltage. The normal corona sound was preceded by a characteristic intermittent sound. The rod was removed and on examination found to have a slender fiber about $1.5 \mathrm{~mm}$ long sticking out perpendicular to the surface of the rod. After removing this fiber and polishing the rod with chamois skin the corona voltage increased. It should be noted that in some cases for which the intermittent sound preceded full corona sound the voltage recorded for the full sound was only slightly higher than that for the former. The necessary voltage to produce full sound in cases where the intermittent sound occurred first was often considerably lower than the value of voltage for the same rod for conditions such that the initial corona sound was loud and continuous.

In one instance, after replacing the $\mathrm{CaCl}_{2}$ in the drying tube, a considerable portion of $\mathrm{CaCl}_{2}$ dust was evidently blown into the corona voltmeter, for on resuming operations it was found that a proper continuous initial corona sound could not be obtained until the wad of cotton in the air strainer was replaced and the pipes and corona voltmeter were blown out with compressed dry air to remove the dust.

The combined presence of humidity and soiled condition of the surface causes greater lowering of corona voltage than is produced by either separately. This combined effect will be discussed under the effect of humidity.

Further evidence of the importance of surface condition was obtained by cleaning a rod in the usual manner and then carefully fouling it by spraying with a solution of two drops of machine oil 
in $50 \mathrm{ml}$ of C. P. high-test gasoline. The gasoline, after evaporating, left a coating of cylinder oil having an average thickness of 60 molecules. Curve $f$ of Figure 8 was then taken and did not coincide with the corresponding curve $e$ for the cleaned rod. The shapes of these two curves were sufficiently different to confirm the belief that discrepancies between values corrected for humidity for about the same pressures taken at different times may well have been the result of differences in surface condition. As an example of such deviations in the case of rod $D$, we have plotted Figure 8. It should be noted that several series of points, the rod not being disturbed during any given series, lie very close to smooth curves $a, b, c$, etc., while different series are not in close agreement. Curve $b$ was taken at a temperature of about $17^{\circ}$ C., which is somewhat lower than the usual $25^{\circ} \mathrm{C}$., the temperature of the set of runs for curve $a$.

Dubois (10), working with continuous voltages and at much lower pressures (0 to $2 \mathrm{~cm} \mathrm{Hg}$ ), observed that fouling the cathode surface with minute amounts of impurities produced a difference of as much

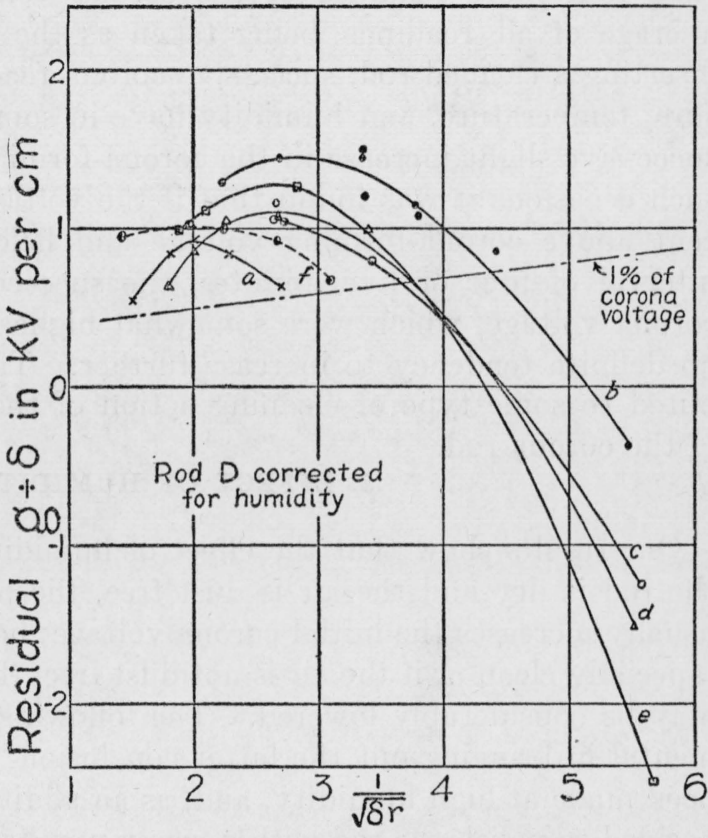

Fig. 8.-Residual values of $g / \delta$ as a function of $1 / \sqrt{\delta r}$ for $\operatorname{rod} D$

The failure of these curves (taken on different days) to coincide is presumably because of changes in surface condition of the rod. Curve $f$ was taken after intentionally coating the rod with a very thin film of oil

as 40 per cent in the disruptive voltage and that this difference diminished decidedly with pressure. Extrapolation of one of his curves to higher pressures indicates that the effect should still be significant in view of the accuracy of our observations and may explain the failure of repeated observations to agree closely. His method of surface cleaning by heating the electrode to a red or white heat is not practicable with the present corona voltmeter equipment but certainly has its advantages. It was not felt when this research was in progress that such minute quantities of soiling material as he used (for example, 1 microgram of $\mathrm{KCl}$ per square centimeter of 
surface) could have any effect, for the degree of polishing of the rod surface is in no wise comparable to the thickness of such a film.

If a carefully cleaned rod be inserted in the corona voltmeter containing dust-free air, the voltage may be raised gradually until corona begins. If the voltage is now lowered until corona ceases and then raised again until corona is detected, the two measurements, all other conditions remaining the same, usually agree to within a few hundredths of 1 per cent. In some instances greater differences have been observed. In such cases it was customary to take at least four more readings of the corona voltage for each run, the average of all readings being taken as the corona voltage. After inserting a cleaned rod, successive corona readings at the same pressure, temperature, and humidity have in some instances indicated a successive slight increase in the corona-forming voltage. On several such occasions it was found that if the voltage was "raised a few per cent above corona-forming voltage and held at that value for an interval of four or five minutes, the succeeding readings of initial corona voltage, which were somewhat higher than the first, showed no definite tendency to increase further. This effect may be attributed to some type of cleaning action of the corona at the surface of the corona rod.

\section{EFFECT OF HUMIDITY}

Our results show that the effect of humidity may be twofold. If the rod is dry and the air is dust free, the presence of water vapor usually increases the initial corona voltage; however, if the rod is not especially clean or if the air is not dust free, the initial corona voltage may be considerably lowered. The following experience was instrumental in bringing out the latter conclusion. Several runs had just been made at high humidity, and, as an additional precaution, it was decided to polish the rod with a newly purchased chamois skin before taking further runs at reduced humidity. On attempting to check the runs at high humidity after polishing with the new chamois skin and prior to reducing humidity, it was found that the corona voltage was from 2 to 5 per cent lower than for corresponding runs previously taken. The humidity was then reduced, and for one-fourth of the moisture present the corona voltage was found to be but 0.5 per cent lower than for values obtained previously for about the same humidity. The rod was therefore removed, polished slightly with crocus paper, washed and dried with redistilled ethyl alcohol, then rubbed with the chamois skin, which in the interim had been washed with ether and redistilled ethyl alcohol to remove all traces of tanning oil and tanning products. A new run was taken with almost dry air, and the corona voltage was found to nearly coincide with earlier values for dry air. The lowering in corona voltage noted above is ascribed to the combined presence of water and surface con- 
tamination. Undoubtedly the newly purchased chamois skin contained tanning products that soiled the rod surface when the chamois skin was used in an attempt to further clean the surface. This experience led to the above-described definite procedure in cleaning corona rods before use.

In order to ascertain the effect of high humidity with dust present on the corona rod, rod $K$ was left in the machine shop on a high shelf for several days after it had been previously cleaned. It was inserted in the corona voltmeter without disturbing the accumulated dust on its surface and corona readings were made for several different pressures. The air within the corona voltmeter was then humidified and additional runs were made. In the latter case there were, roughly, six times as many water molecules present in the air, according to dew-point measurements, as in the former case, and the corona voltage was found to be approximately 20 per cent lower. The rod was then cleaned, and the corona voltage was found to be about 30 per cent higher than in the last case; in other words, for the dusty rod in dry air the corona voltage was about 10 per cent lower than for the clean rod in dry air.

Rod $C$ was cleaned and inserted in the corona voltmeter, and a run was made with the air fairly humid. The rod was then soiled by rubbing it with a sweaty but otherwise fairly clean hand. As a result the corona voltage was found to be about 6 per cent lower. The air was then dried and the corona voltage increased, but was still slightly lower than the original dry-air value. The same rod, after cleaning, was wiped with a hand covered with machine oil. Subsequently the corona voltage was found to be several per cent lower.

Humidity runs were made on rods $A, D$, and $K$ at room temperature and are given in Figure 9. It is much less difficult to control humidity and air-temperature difference at room temperature than at higher or lower temperatures. Figure 9 is plotted using the dry-air corona values as a basis of comparison. The differences between dry-air values and values taken at different humidities have been plotted for several values of $1 / \sqrt{\delta r}$. In order to avoid confusion, all points have not been plotted on the curves. Points have been plotted in the case of $\operatorname{rod} A$ for $1 / \sqrt{\delta r}=4.20$, rod $D$ for $1 / \sqrt{\delta r}=2.69$, and rod $K$ for $1 / \sqrt{\delta r}=1.45$. In the case of rods $A$ and $K$ the plotted points are seen to be fairly close to the curves. Each set of points represents a set of humidity runs taken without removing or handling the cleaned rods after their insertion. In the case of rod $D$ it is seen that the points are well scattered on either side of the plotted curve. This group of points represents several sets of runs, between which the rod had been removed, stored, or cleaned. This scattering of points is ascribed to the differences in surface condition. In the case of the other curves plotted on Figure 9 the points fell uniformly on smooth 
curves, and if the surface of the rod had not been disturbed the corona voltage would repeat closely on returning to a value of humidity previously observed. Where several sets of runs with the same rod for the same value of $1 / \sqrt{\delta r}$ were made at different times, scattering was observed, as illustrated by the plotted points of $\operatorname{rod} R$.

The values plotted in Figure 9 were obtained with certain selected rods and at selected values of $1 / \sqrt{\delta r}$ in the attempt to derive a basis for correcting observations on all the rods to the dry-air condition. Since for most of these runs the humidity was quite low, it was thought that this basis of correction would be sufficient. In order

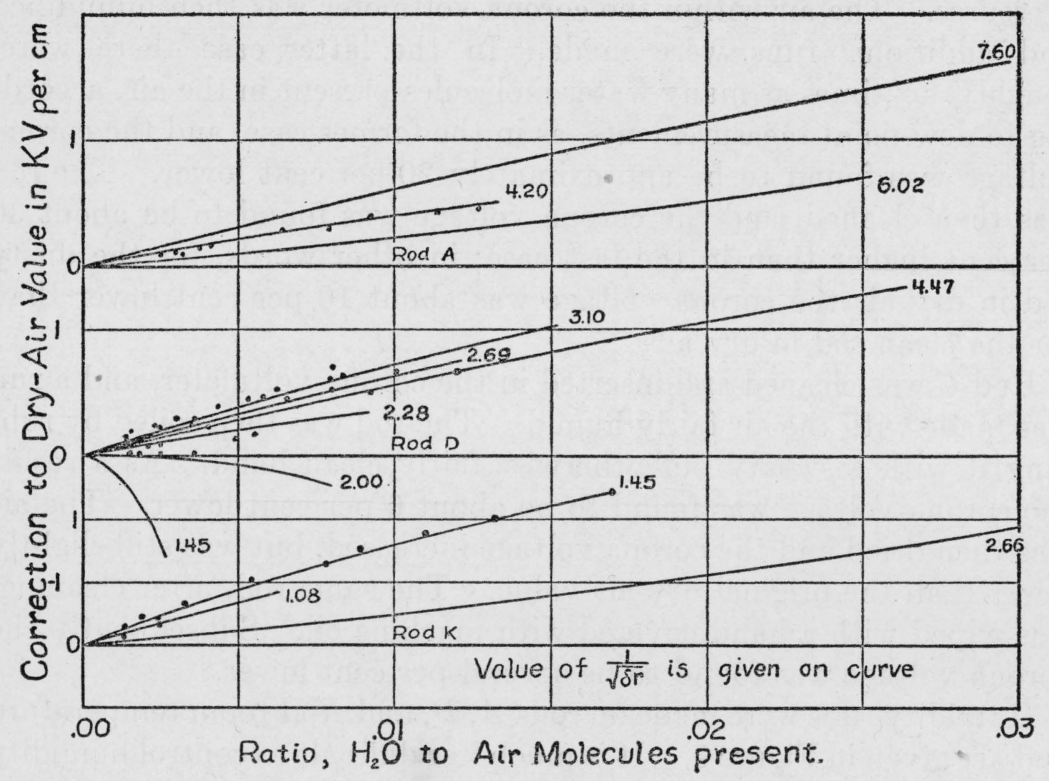

Fig. 9.-Effect of humidity on $g / \delta$ for various rods and density factors

to use the observed data for this purpose, several sets of derived curves were plotted, giving the humidity correction in per cent corona voltage plotted against $1 / \sqrt{\delta r}$ for a definite ratio of water to air molecules present. Interpolation between these curves serves as a means of obtaining corrections to be applied to the various rods. Unfortunately, this method of humidity correction is not entirely satisfactory. Its application to earlier runs in which less careful cleaning methods were used was found to cause successive points which originally fell on smooth curves to depart appreciably from smooth curves. It therefore seems desirable in future work to take sets of humiditycorrection runs for each rod and possibly at several different temperatures. Our present program would not permit this investigation.

Although the previously mentioned special precautions were employed in cleaning corona rods before use, the work of Dubois and our own observations with deliberately fouled rods suggests that 
possibly even our careful cleaning was insufficient. If such be the case, our disagreement between runs on different-sized rods may be explained on the basis that the rods were differently soiled and that the humidity effect at a given air density depends on the nature of the fouling of the surface. It is interesting to note that under our experimental conditions the correction required as a result of humidity has been as much as 3 per cent in extreme cases. By using reasonable precautions to control humidity the correction could be greatly reduced.

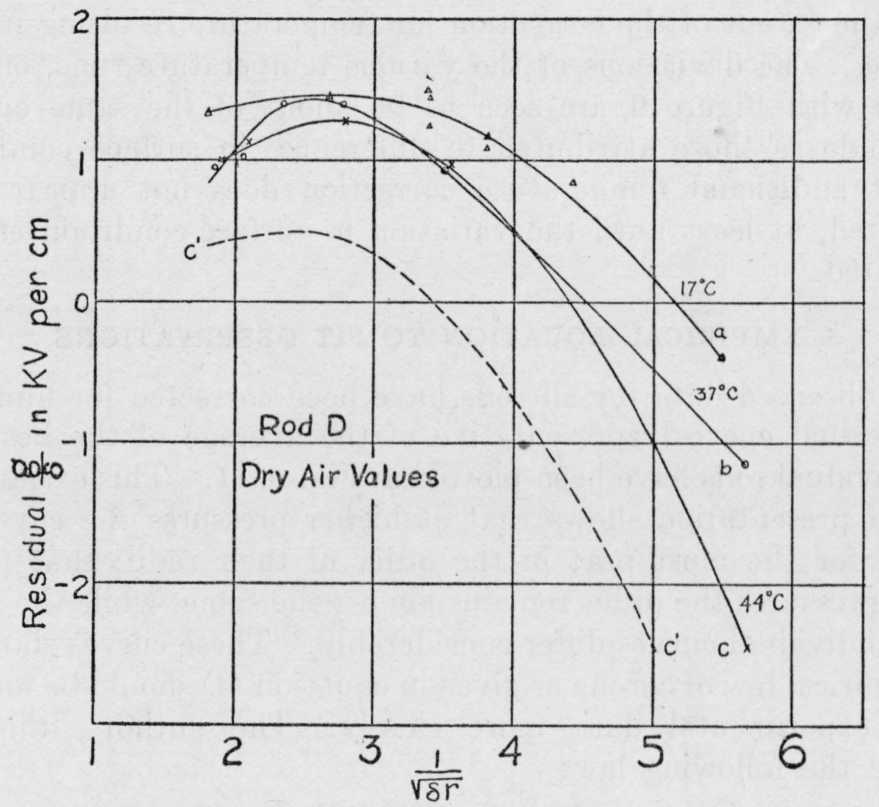

FIG. 10.-Residual values of $g / \delta$ as a function of $1 / \sqrt{\delta r}$, showing the effect of temperature

Curve $c^{\prime}$ is plotted from the data of Curve $c$, but with the temperature arbitrarily taken as $25^{\circ} \mathrm{C}$. instead of $44^{\circ} \mathrm{C}$.

\section{EFFECT OF CHANGE IN TEMPERATURE}

Temperature enters the law of corona through the density factor $\delta$. In operating the corona voltmeter it is not desirable to alter the temperature as a means of increasing the range of the instrument, for it is much simpler to alter the pressure. In fact, it is desirable to use the corona voltmeter at room temperature, so that corrections would need to apply only for a small range of temperature. Others have investigated the use of the density-factor correction and have found it to agree within experimental accuracy within the range with which we are concerned. It seemed desirable, however, in view of our increased accuracy of measurement, to make several"sets of runs at temperatures other than room temperature to determine whether further corrections should be applied. Results of such runs, corrected 
as usual for humidity, were plotted as residuals for rods $C, D, J$, and $K$, of which the runs on rod $D$ alone appear in Figure 10. Only these runs are presented, because they alone were taken consecutively without removal or handling of the rod and should show the effect of temperature only. Curves $a, b$, and $c$ are for different temperatures, while $c^{\prime}$ represents the curve which would have been obtained if the data of curve $c$ had been plotted on the assumption that they corresponded to a temperature of $25^{\circ} \mathrm{C}$. instead of their actual temperature of $44^{\circ} \mathrm{C}$. The difference between $c$ and $c^{\prime}$, therefore, shows the extent of the correction for temperature resulting from its use in $\delta$. The deviations of the various temperature runs, on comparison with Figure 9, are seen to be about of the same order of magnitude as those attributed to differences in surface conditions, so that additional temperature correction does not appear to be warranted, at least until the variation in surface-condition effect is eliminated.

\section{EMPIRICAL EQUATION TO FIT OBSERVATIONS}

The observed data for all rods have been corrected for humidity, and residual curves representative of the average of the best data for individual rods have been plotted in Figure 11. This exaggerated basis of presentation shows that at higher pressures the curves are located for the most part in the order of their radii; that for the lowest pressures the order remains almost the same; while the shapes of the individual curves differ considerably. These curves show that the empirical law of corona as given in equation (4) should be modified to fit experimental data more closely. The authors, therefore, propose the following law:

$$
g=A \delta+B \sqrt{\delta / r}-C / r
$$

This equation fits the experimental data corrected for humidity more closely than equation (4). It is shown plotted as curve $R$ in Figure 11, using $\mathrm{A}=27.95, \mathrm{~B}=11.18$, and $\mathrm{C}=0.365$. The same equation (6) has been plotted in Figure 12, and a large number of points observed under good experimental conditions have been plotted in a self-explanatory manner. Points that fall within the envelope have not been designated on the graph. This graph illustrates the accuracy of our proposed empirical equation in expressing the law of corona as determined experimentally. Figure 12 should bring out the fact that the empirical relation above is not all that can be desired in the way of a law to express corona formation, for it indicates that some further function of the radius $r$ might bring the experimental observations into closer agreement. 
Figure 13 represents a plot of residuals against radius of rod for constant values of $\delta$ and is derived from the experimental curves of Figure 11. These point-to-point plots should probably be smooth curves, and the fact that they are not may be because of improper humidity correction and slightly differing surface conditions.

\section{A NOMOGRAM THAT SIMPLIFIES VOLTAGE DETERMINATION}

Although the primary objects of this research have been to determine accurately the law of corona and the effect of humidity on the value of corona-forming voltage, it was also felt that for factory test-room use a procedure should be devised to give the result (crest voltage at the terminals of the corona voltmeter) from the pressure

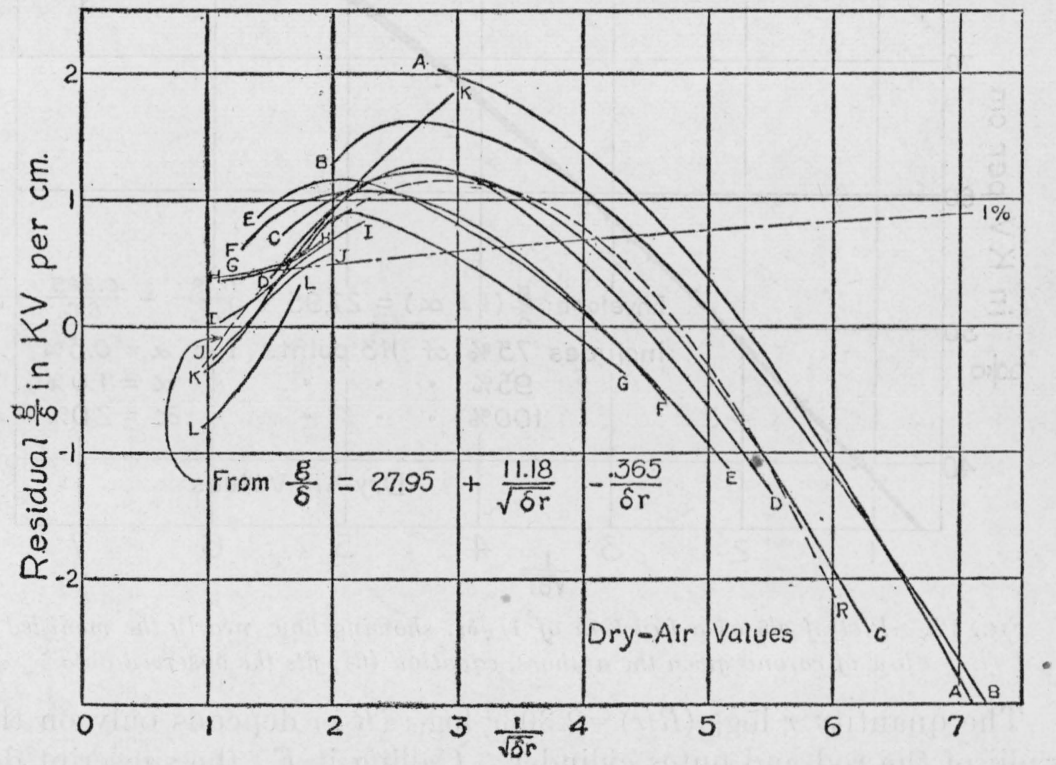

FIG. 11.-Residual values of $g / \delta$ as a function of $1 / \sqrt{\delta r}$, for all the rods, after correcting for humidity

Dotted line is plotted from law of corona as modified by the authors to represent as well as possible the average performance of all the rods

and temperature readings quickly and without any computation. If we denote the crest voltage applied to the corona voltmeter by $V_{\mathbf{c}}$, the critical gradient at the surface of the rod by $g$, and the radii of the rod and surrounding cylinder by $r$ and $R$, respectively, we have the relation

$$
g=\frac{V_{\mathrm{e}}}{r \log _{\mathrm{e}}(R / r)}
$$

from which the crest voltage is

$$
V_{\mathrm{e}}=g r \log _{\mathrm{e}}(R / r)
$$


The empirical equation for the critical gradient is

$$
g=A \delta+B \sqrt{\delta / r}-C / r
$$

Inserting this value in equation (8), we have

$$
\begin{aligned}
V_{\mathrm{c}} & =(A \delta+B \sqrt{\delta / r}-C / r) r \log _{\mathrm{e}}(R / r) \\
& =(A \delta+B \sqrt{\delta / r}-C / r) 2.303 r \log _{10}(R / r)
\end{aligned}
$$

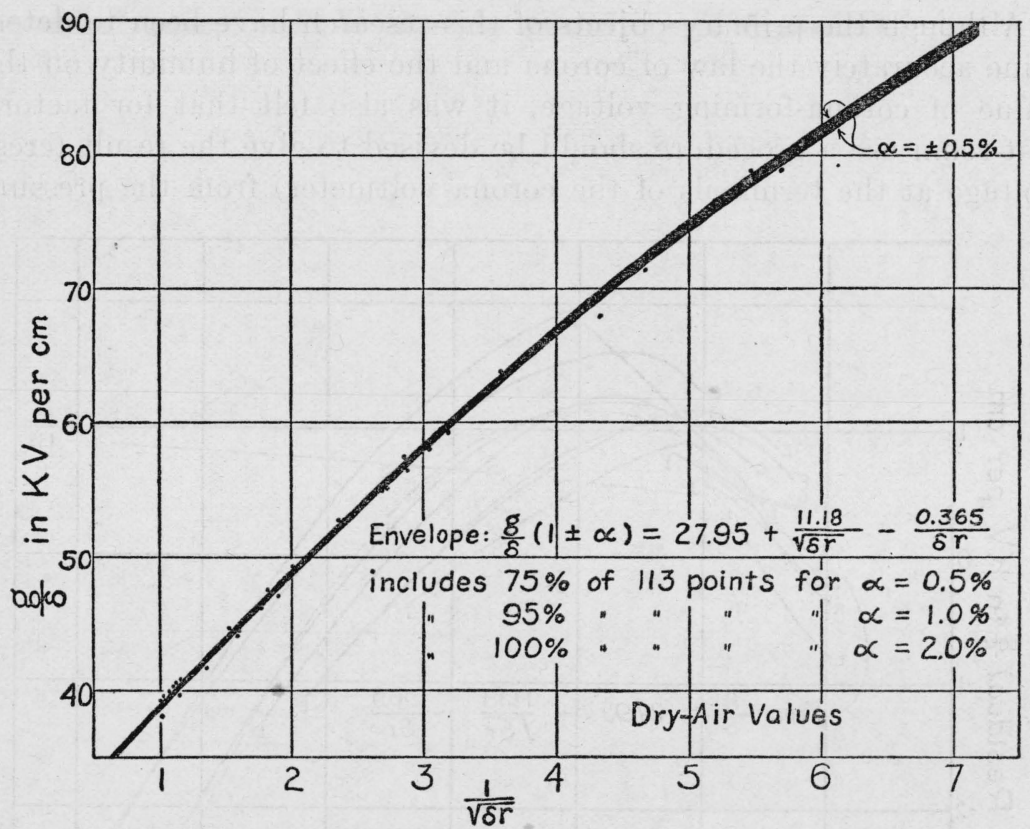

FIG. 12.-Plot of $g / \delta$ as a function of $1 / \sqrt{\delta r}$, showing how nearly the modified law of corona given the authors, equation (6), fits the observed data

The quantity $r \log _{\mathrm{e}}(R / r)=2.303 r \log _{10}(R / r)$ depends only on the radii of the rod and outer cylinder. Calling it $F_{\mathrm{r}}$, the subscript denoting the radius of the rod, equation (11) becomes

$$
V_{\mathrm{c}}=F_{\mathrm{r}}\left(A \delta+\frac{B}{\sqrt{r}} \sqrt{\delta}-C / r\right)
$$

The coefficient of $\sqrt{\delta}$ in equation (12) is constant for any given rod. Denote it by $D_{\mathrm{r}}$, the subscript being the radius of the rod. Then

$$
\begin{aligned}
V_{\mathrm{c}} & =F_{\mathrm{r}}\left(A \delta+D_{\mathrm{r}} \sqrt{\delta}-C / r\right) \\
& =F_{\mathrm{r}}\left(3.921 A P / T+D_{\mathrm{r}} \sqrt{3.921 P / T}-C / r\right) \\
& =M_{\mathrm{r}}(P / T)+N_{\mathrm{r}} \sqrt{P / T}-Q_{\mathrm{r}}
\end{aligned}
$$

where

$$
\begin{aligned}
M_{\mathbf{r}} & =3.921 \times 2.303 A r \log _{10}(R / r) \\
& =9.03 A r \log _{10}(R / r)
\end{aligned}
$$




$$
\begin{aligned}
N_{\mathrm{r}} & =\sqrt{3.921} \times 2.303 B \sqrt{r} \log _{10}(R / r) \\
& =4.56 B \sqrt{r} \log _{10}(R / r) \\
Q_{\mathrm{r}} & =F_{\mathrm{r}} C / r=2.303 r \log _{10}(R / r) \times C / r \\
& =2.303 C \log _{10}(R / r)
\end{aligned}
$$

For a given cylinder the values of $M_{\mathrm{r}}, N_{\mathrm{r}}$, and $Q_{\mathrm{r}}$ for the rods to be used may be computed and tabulated. With a given rod in use,
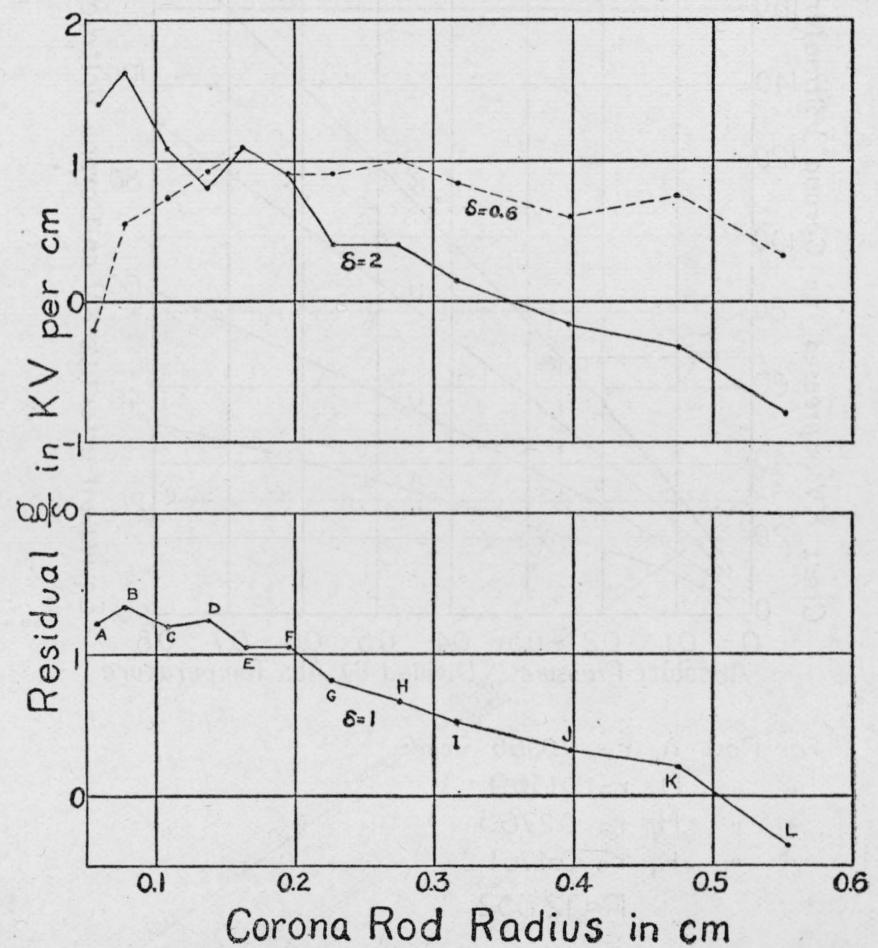

FIG 13.-Plot of residual values of the quantity $g / \delta$ as a function of the radius of the corona rod, for three values of density factor $\delta$

the crest voltage at the instrument terminals may then be found by substituting in equation (14) the quantity $P / T$; that is, the quotient of the observed absolute pressure divided by the observed absolute temperature. The form of equation (14) adapts it to convenient calculations with a slide rule.

The next step in reducing the labor of computation is to plot a family of curves giving $V_{\mathrm{c}}$ as a function of $P / T$, having a curve for each rod used. This reduces the calculation to merely division of observed absolute pressure by observed absolute temperature. Such a family of curves is given in the upper part of Figure 14. 
All computation may be avoided by combining with the above family of curves a nomogram of the form shown in the lower part of Figure 14. It is necessary only to lay a straightedge so that it crosses the temperature and pressure scales at points corresponding to the observed temperature and pressure. It then crosses the "quotient" scale at the corresponding value of $P / T$. The ordinate of this abscissa, read on the curve for the rod in use, gives crest voltage on

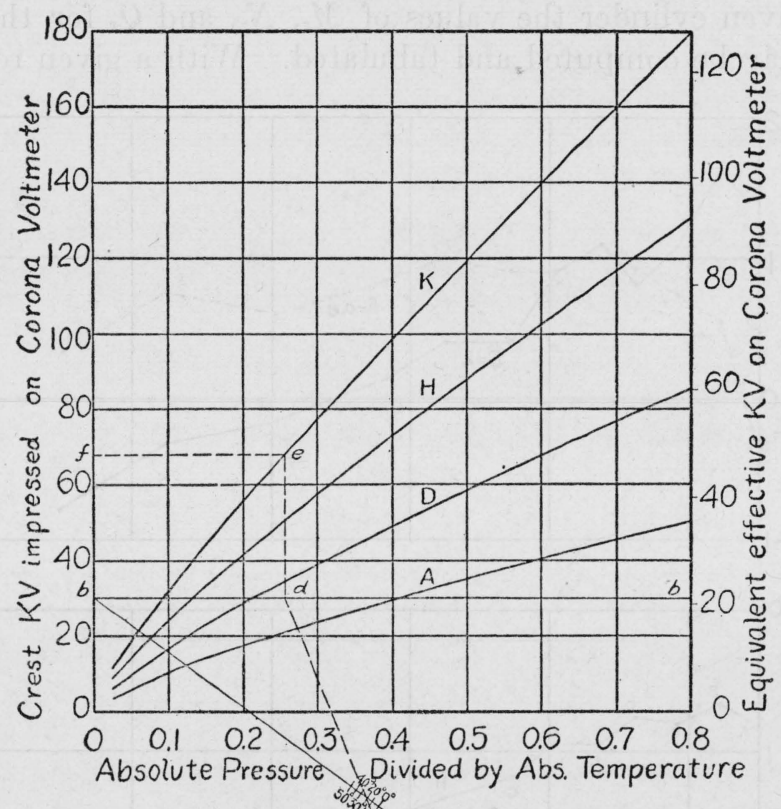

$$
\begin{aligned}
\text { For Rod } A, r & =0.0566 \\
" \text { " } D, r & =0.1369 \\
" \text { " H, } r & =0.2765 \\
K, & =0.4761 \\
& R=12.352
\end{aligned}
$$

$$
\begin{aligned}
& 200180160140 \quad 120 \quad 100 \quad 80 \quad 60 \quad 40 \quad 20 \\
& \text { Absolute Pressure in cm of Mercury }
\end{aligned}
$$$$
\begin{array}{llllllllllllll}
\hline \text { ic } & 180 & 160 & 140 & 120 & 100 & 80 & 60 & 40 & 20 & 0
\end{array}
$$

FIG. 14.-Nomogram for use with the corona voltmeter to obtain values of crest voltage (or equivalent sine-wave effective voltage) without any calculation

the left-hand ordinate scale and equivalent sine-wave effective voltage on the right-hand scale.

In making this nomogram one may take advantage of the fact that the relative change in magnitude of the absolute temperature is small compared with the relative change in absolute pressure and in the quotient $P / T$. The quotient scale and the pressure scale may therefore be arbitrarily laid out with divisions of uniform length 
throughout, leaving all of the nonuniformity to occur in the temperature scale.

A modification of this method, suggested by R. H. Marvin, consists in using a nomogram of which the top and bottom lines are the pressure scale and the temperature scale, while between them is a family of scales, one for each wire to be used, on which the crest voltage is read.

The scales of either nomogram do not need to be marked in terms of the absolute pressure and temperature upon which they are based but may be marked in ${ }^{\circ} \mathrm{F}$. or ${ }^{\circ} \mathrm{C}$. (or both, if desired), and in any pressure units, such as in centimeters of mercury above atmospheric pressure, in pounds per square inch gauge pressure, etc.

\section{ADVANTAGES AND DISADVANTAGES OF THE CORONA VOLTMETER AND SUGGESTIONS FOR ITS IMPROVE- MENT}

As a device for measuring the crest value of alternating voltage, the corona voltmeter may be compared with the sphere gap. In 1925 one of the authors had occasion to review sphere-gap literature in connection with work on the International Critical Tables and was forced to conclude that the average accuracy to be expected from individual sphere-gap measurements was not much better than \pm 5 per cent. Aside from inherent time lag, some portion of this inaccuracy was undoubtedly a result of insufficient attention to the sphere gap itself and to its surroundings. A graph in an article by Hayden and Eddy (11) shows 500 breakdowns for air between 2.54 $\mathrm{cm}$. spheres. They conclude: "The maximum error of test does not exceed 4 per cent, and the average error is 1 per cent. In the average of six successive tests the maximum error decreases from 4 per cent to 2.9 per cent and the average error decreases from 1 per cent to 0.6 of 1 per cent." In the case of the corona voltmeter, it has already been noted that successive readings of the same point usually vary less than 0.05 of 1 per cent. Agreement for the same rod subsequent to handling and cleaning is considerably poorer, and individual points may deviate from the average curve by as much as 1 per cent. This difference is probably the result of alteration of the surface condition. If measurements are made over a period of a few hours, during which time nothing occurs to change the surface condition of the rod or the humidity, the individual points may be expected to lie within 0.1 per cent of a smooth curve for that rod.

It should be remembered that the concentric-cylinder construction of the corona voltmeter eliminates one indeterminate disturbing element encountered in the noninclosed sphere gap, namely, that arising from the proximity of other objects. Properly designed and inclosed, the corona voltmeter is unaffected by surrounding objects. 
The operation of either the corona voltmeter or the sphere gap consumes only a negligible amount of volt-amperes which is purely reactive until corona starts or the gap breaks down. At breakdown the sphere-gap transformer circuit is likely to oscillate in a manner that is often injurious to insulation unless sufficient resistance is placed in series with the gap. Since the voltage applied to the corona voltmeter should never be raised to spark over, transients resulting from its use are of no consequence, and the power is negligibly small even when spark over is impending.

In corona voltmeters it has been customary to use an outer cylinder of fixed diameter and to alter the inner cylinder or rod diameter and air pressure as a means of changing voltage range. These methods of change in range for the corona voltmeter are not as convenient as the change in range of the sphere gap by altering its length.

It is well known that sphere-gap spark over may be readily observed either visually or aurally. Observations of initial formation of corona, however, make auxiliary apparatus desirable or even necessary, except in the case of the visual method, which is unsatisfactory for laboratory or factory use. Aural detection is applicable to advantage, except in very noisy surroundings. We found this method quite satisfactory for use in our laboratory in which the supply motorgenerator set was running, if we used airplane-head-set phones in conjunction with a resistance-coupled amplifier. Unless extraneous noises striking the microphone are largely eliminated, the advantage of amplification may be considerably reduced. The ionization method, although readily applicable, requires either one more observer, as in the case of the visual method, or additional apparatus.

Spark over within the corona voltmeter produces a deafening noise that may not be tolerated in the case of aural detection, and, in addition, it is injurious to the surface of the corona rod. On several occasions spark over occurred while readings were being taken with the larger rods above atmospheric pressure. Since it is highly desirable from an observational viewpoint to have the voltage difference between initial formation of corona and spark over large in order to avoid accidental spark over, it appears desirable that the regions over which corona and spark over occur separately be determined. Our observations indicate that at atmospheric pressure the ratio of the cylinder radii should be greater than $20: 1$ and that the region previously referred to is a function of air pressure or density as well as humidity. Further study of these limitations of the size of the corona voltmeter should prove interesting.

It should not be overlooked that greater accuracy in crest-voltage measurements with the sphere gap might be obtained by inclosing the gap in a container which would permit the control of air pressure and humidity. With any practicable size of inclosure, the effect of its 
walls would be to modify the electrostatic field and, consequently, to alter the law of spark over as a function of the gap between the spheres. This law would have to be redetermined for definite relative dimensions of inclosure and spheres and a given arrangement of these parts.

If the corona voltmeter is to share the duties of the sphere gap or replace it as a commercial measuring device, in addition to its superior accuracy and freedom from tendency to produce circuit disturbances it must afford comparative ease of operation.

The present corona voltmeter offers room for improvement from the standpoint of design. From Figure 14 one may observe that two rods, a large one and a small one, would be sufficient to cover the entire range covered by the larger number of rods used in this investigation, provided the pressure may be varied over a sufficiently wide range. The use of a wide pressure range requires that the pressure container and insulating bushing be mechanically independent of the corona rod, so that alteration in pressure will cause no change in relative location of the corona rod and the outer cylinder. (In the present instrument we found it necessary to introduce a tension spring to hold the rod taut and thus counteract a shift of the metal fittings of the insulating bushing.) If rods must be changed, a simpler, quicker, and more direct method of insertion of a cleaned rod is highly desirable.

The thermal design of the corona voltmeter could be improved if it is desired to use it at its highest attainable precision. Since it would usually be operated at room temperature, the use of a circulating fan to keep the air well stirred would be sufficient, and the precision which one desired would determine whether measurement of air temperature would be desirable or simply measurement of the container temperature would be sufficient. The effect of temperature difference on the accuracy of measurement is discussed in the appendix.

The method of pressure measurement used in these experiments is too complicated for ordinary work. If the use of commercially available pressure gauges would not provide sufficient accuracy for the work, a sylphon type of instrument might be designed for the purpose that could be calibrated directly in absolute pressure. If it were desired to apply humidity correction rather than avoid the necessity for it by providing thoroughly dry air, a dew-point hygrometer might well be located in the inclosed chamber. It might be convenient to provide air circulation for the corona chamber by circulating the air by a small fan through an auxiliary smaller chamber in which pressure-measuring, humidity-measuring, and temperaturemeasuring instruments, as well as apparatus for air drying or humidifying could be located. By removing the air from the top of the 
corona voltmeter, passing it through the auxiliary chamber and then back in to the bottom of the corona voltmeter, good air circulation could be provided with minimum alteration of the corona chamber itself.

By properly locating the measuring and detecting instruments and controls of the corona voltmeter it should be possible for a single observer to carry on all the manipulations needed to determine crest voltage.

It has already been emphasized that future investigations relative to increase in accuracy and repeatability of the corona voltmeter should begin with a careful study and control of the condition of the surface of the rod. The condition of the surface of the outer cylinder is presumably of lesser importance, because the gradient at its surface is much lower. A more thorough study of the mechanism of corona formation is suggested.

\section{ACKNOWLEDGMENTS}

In this publication reference has undoubtedly been made to authors whose investigations have been antedated by other investigations, and statements have been made without direct reference to prior work of any particular investigator, so that in reading this work one should not overlook the fact that other works besides those mentioned in the bibliography have been freely examined and available useful material has been used ad libitum.

The authors are indebted to Dr. J. B. Whitehead, of Johns Hopkins University, for the loan of the corona voltmeter and advice relating to its use and improvement, and to his associates, Dr. W. B. Kouwenhoven and Dr. F. W. Lee, for valuable suggestions during the progress of the investigation. The mechanical alterations in the apparatus were executed in an excellent manner by J. M. S. Kaufman, of the instrument shop of this bureau. The advice of Dr. F. B. Silsbee, together with his help and that of F. K. Harris, A. B. Lewis, and others of the bureau staff in making observations and computing results have been invaluable.

\section{APPENDIX}

\section{EFFECT OF ERRORS IN OBSERVED DATA}

The individual effects of small relative errors in the observed data were investigated analytically to determine the relative accuracy required in each in order to attain a given degree of accuracy in the measured value of crest voltage. Assuming the constants $A, B$, and $C$ as known, the four observed quantities are $r$ and $R$, the radii of the rod and cylinder, respectively, and $P$ and $T$, the absolute pressure and temperature of the air. 
If we differentiate equation (10) with respect to $r$ and divide the result by the right-hand member of equation (10) and simplify, the following result is obtained:

$$
\frac{d V_{\mathrm{e}}}{V_{\mathrm{c}}}=\frac{\left(A \delta+\frac{B}{2} \sqrt{\delta / r}\right) \log _{\mathrm{e}}(R / r)-(A \delta+B \sqrt{\delta / r})-C / r}{(A \delta+B \sqrt{\delta / r}) \log _{\mathrm{e}}(R / r)+C / r \log _{\mathrm{e}}(R / r)} \cdot \frac{d r}{r}
$$

Figure 15 shows the manner of variation of the coefficient of $d r / r$ as a function of $r$. It will be seen that the relative error in the crest voltage is always less than the relative error in the radius of the rod; that it depends on this radius and that of the surrounding cylinder, as well as on the pressure and the temperature.

It should be pointed out that, since the error in measuring the diameter of a rod by usual means (such as a micrometer caliper) tends

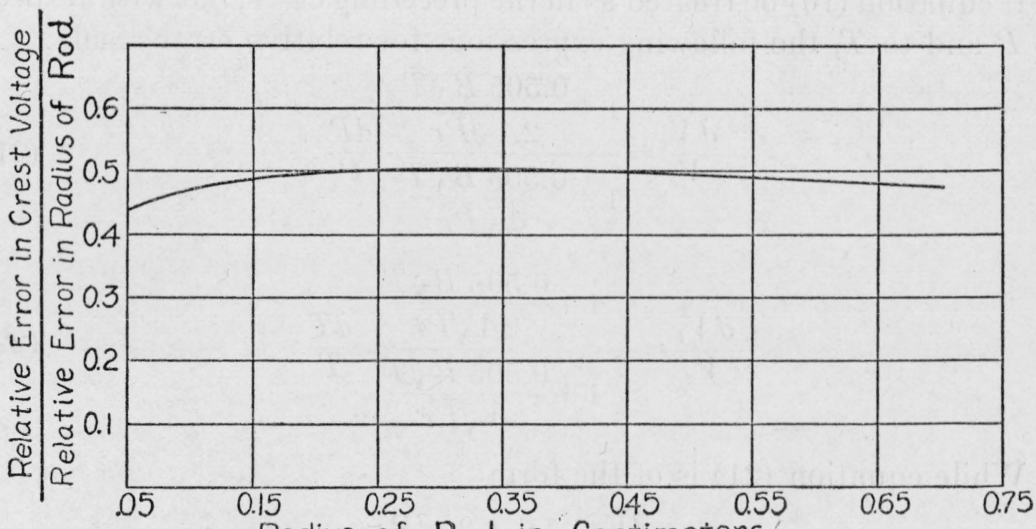

Radius of Rod in Centimeters

FIG. 15.-Curve showing effect on computed crest voltage of an error in measuring the diameter of the rod

toward a constant absolute amount, the relative error will tend to increase as the radius decreases. It is thus important not to use rods any smaller than is necessary.

Treating equation (10) in like manner, but differentiating with respect to $R$, gives

$$
\frac{d V_{\mathrm{e}}}{V_{\mathrm{e}}}=\frac{1}{\log _{\mathrm{e}}(R / r)} \cdot \frac{d R}{R}
$$

In this case the relative error in crest voltage is a simple function of the ratio $R / r$ and, unlike the preceding case, is independent of $A, B$, $C, P, T$, and the absolute value of $r$. In practice $R / r$ would usually be from 10 to 100 , so that the relative error in crest voltage resulting from an error in $R$ will always be appreciably less than $d R / R$, as shown by the following values:
$R / r=10$
30
40
50
$\begin{array}{ll}75 & 100\end{array}$
200
$d V_{\mathrm{e}} / V_{\mathrm{e}}=0.43$
0. 33
0. 29
0. 28 0. 26
$0.23 \quad 0.22$
$0.19 \times d R / R$ 
Relative errors in the measurement of the radius of the surrounding cylinder, therefore, have much less effect on the measurement of crest voltage than the same relative errors in the measurement of the radius of the rod. The case is much more favorable for accurate measurement of the radius of the cylinder because of the greater magnitude to be dealt with. Hence it is unnecessary to have a high degree of precision of form of the surrounding cylindrical surface, or to have the rod exactly coaxial with it, since either a slight displacement of the rod from the coaxial position or a slight ellipticity of the cylindrical surface may be considered equivalent to a small change in $R$. Furthermore, the gradient being very much lower at the surface of the cylinder than at the surface of the rod, small irregularities of surface, dust, and dirt, which must be carefully avoided on the latter, produce a negligible effect on the former.

If equation (10) be treated as in the preceding cases, but with respect to $P$ and to $T$, the following expressions for relative error result:

$$
\begin{gathered}
\frac{d V_{\mathrm{c}}}{V_{\mathrm{c}}}=\frac{1+\frac{0.505 B \sqrt{T}}{2 A \sqrt{P r}}}{1+\frac{0.505 B \sqrt{T}}{A \sqrt{P r}}} \cdot \frac{d P}{P} \\
\frac{d V_{\mathrm{c}}}{V_{\mathrm{c}}}=-\frac{1+\frac{0.505 B \sqrt{T}}{2 A \sqrt{P r}}}{1+\frac{0.505 B \sqrt{T}}{A \sqrt{P r}}} \cdot \frac{d T}{T}
\end{gathered}
$$

While equation (21) is of the form

$$
\frac{d V_{\mathrm{e}}}{V_{\mathrm{c}}}=\frac{1+a / 2}{1+a} \cdot \frac{d P}{P}
$$

and the right-hand member of equation (22) differs from that of equation (21) only in sign, it is not practicable to expand the coefficient of $d P / P$ or that of $d T / T$ into a series, on account of the large variation in the value of $a$ arising from the term $\sqrt{P r}$. With pressures of only a few cm of mercury and rods a fraction of a $\mathrm{cm}$ in diameter, $a$ will be greater than unity, while for a rod $1 \mathrm{~cm}$ in diameter and an absolute pressure of $152 \mathrm{~cm}$ of mercury, $a=0.22$. Hence, it is preferable to use the expressions (21) and (22).

It is evident from equation (22) that the quantity which may be termed the temperature coefficient of the electric strength of air between concentric cylinders is dependent upon the geometry of the bounding surfaces, since it varies with the radius of the rod as well as with the absolute pressure and temperature. The range of variation of this temperature coefficient is, however, not large for practical values of the variables. It may be shown that, as an approximate rule, the relative error in crest voltage may be taken as numerically 
equal to 0.8 times the relative error in either temperature or pressure. For example, an error in temperature of $1^{\circ} \mathrm{C}$. when the air temperature is $25^{\circ} \mathrm{C}$. $\left(=298^{\circ} \mathrm{Abs}.\right)$ produces an error of $0.8 \times(1 / 298)=0.0027=$ 0.27 per cent in the crest voltage, and an error of $1 \mathrm{~mm}$ in measuring a pressure of $76 \mathrm{~cm}$ results in an error of $0.8 \times(1 / 760)=0.0011=0.11$ per cent.

In order to compare the results obtained by using formula (22) with values of the per cent change in gradient per ${ }^{\circ} \mathrm{C}$. as determined by previous experimenters, the data given by Whitehead and Fitch (3) in their Table III were used. These relate to three corona rods of $0.12,0.16$, and $0.20 \mathrm{~cm}$ radius, the gradient having been determined for each at three temperatures approximately $4^{\circ}, 24^{\circ}$, and $52^{\circ} \mathrm{C}$. The values computed from the experimental data and those computed by formula (22) are as follows:

\begin{tabular}{|c|c|c|c|c|c|c|}
\hline \multirow[b]{2}{*}{$\begin{array}{l}\text { From Whitehead and Fitch data } \\
\text { Computed from formula }(22)\end{array}$} & \multicolumn{6}{|c|}{ Per cent change in critical gradient per ${ }^{\circ} \mathrm{C}$. } \\
\hline & $\begin{array}{r}0.28 \\
.25\end{array}$ & $\begin{array}{r}0.24 \\
.25\end{array}$ & $\begin{array}{r}0.31 \\
.26\end{array}$ & $\begin{array}{r}0.24 \\
.26\end{array}$ & $\begin{array}{r}0.31 \\
.26\end{array}$ & $\begin{array}{r}0.27 \\
.26\end{array}$ \\
\hline $\begin{array}{l}\text { Average: } \\
\text { Whitehead and Fitch } \\
\text { Formula }(22)\end{array}$ & $\begin{array}{l}0.275 \mathrm{pe} \\
0.257 \mathrm{pe}\end{array}$ & $\begin{array}{l}\text { cent pe } \\
\text { cent pe }\end{array}$ & ○ $\mathrm{C}$. & & & \\
\hline
\end{tabular}

\section{REFERENCES}

1. Ryan, H. J., Trans. A. I. E. E. 23, p. 101; 1904.

2. Whitehead, J. B., Trans. A. I. E. E. 29, pp. 1159-1187; 1910; 30, pp. 18571887; 1911; 31, pp. 1093-1118; 1912; 34, pp. 1035-1057; 1915.

3. Whitehead, J. B., and Fitch, T. T., Trans. A. I. E. E. 32, pp. 1737-1753; 1913; Whitehead, J. B., and Gorton, W. S., Trans. A. I. E. E. 33, pp. 951-972; 1914; Whitehead, J. B., and Pullen, M. W., Trans. A. I. E. E. 35, pp. 809-833; 1916; Whitehead, J. B., and Brown, W. S., Trans. A. I. E. E. 36, pp. 169-198; 1917; Whitehead, J. B., J. Frank. Inst. 183, pp. 433-451; 1917; Whitehead, J. B., and Isshiki, T., Trans. A. I. E. E. 39, pp. 10571110; 1920; Whitehead, J. B., and Lee, F. W., Trans. A. I. E. E. 40, pp. 1201-1237; 1921; Lee, F. W., and Kurrelmeyer, B., J. A. I. E. E. 44, pp. 16-23; 1925.

4. Peek, F. W., Trans. A. I. E. E. 30, pp. 1889-1965; 1911; 31, pp. 1051-1092; 1912; 32, pp. 1767-1785; 1913; Dielectric Phenomena in High Voltage Engineering, McGraw-Hill; 1920.

5. Farwell, S. P., Trans. A. I. E. E., 33, pp. 1631-1666; 1914.

6. See excellent bibliographies in (12), (13), and (14).

7. Willis, C. H., J. A. I. E. E. 46, p. $272 ; 1927$.

8. Silsbee, F. B., B. S. Sci. Paper No. 516; 1925. See also Orlich, E., and Schultze, H., Archiv f. Elektrotechnik 1, pp. 86, 232; 1912; Kouwenhoven, W. B., Dissertation Elektrotech. Institut Karlsruhe; 1913.

9. Smithsonian Physical Tables, 7th ed., Smithsonian Institution of Washington; 1919.

10. Dubois, E., Ann. de Physique 20, pp. 113-238; 1923.

11. Hayden and Eddy, J. A. I. E. E. 41, p. 397; 1922.

12. Schumann, W. O., Elektrische Durchbruchfeldstärke von Gasen, Julius Springer; 1923.

13. Whitehead, S., Dielectric Phenomena, Ernest Benn (Ltd.); 1927.

14. Loeb, L. B., J. Franklin Inst. 205, p. 305; 1928.

Washington, May 25, 1928. 
